\title{
Numerical analysis of the Rayleigh instability in capillary tubes: The influence of surfactant solubility
}

\author{
D. M. Campana \\ Instituto de Desarrollo Tecnológico para la Industria Química (INTEC), CONICET_Universidad Nacional \\ del Litoral, Güemes 3450-S3000GLN Santa Fe, Argentina and Facultad de Ingeniería, Universidad \\ Nacional de Entre Ríos, Ruta Prov. 11 Km.10—C.C. 57, Suc. 3, E3100, Paraná, Entre Ríos, Argentina \\ F. A. Saita ${ }^{a}$ \\ Instituto de Desarrollo Tecnológico para la Industria Química (INTEC), CONICET-Universidad Nacional \\ del Litoral, Güemes 3450—S3000GLN Santa Fe, Argentina
}

(Received 16 July 2005; accepted 17 January 2006; published online 24 February 2006)

\begin{abstract}
A two-dimensional (2D) free surface flow model already used to study the Rayleigh instability of thin films lining the interior of capillary tubes under the presence of insoluble surfactants [D. M. Campana, J. Di Paolo, and F. A. Saita, "A 2-D model of Rayleigh instability in capillary tubes. Surfactant effects," Int. J. Multiphase Flow 30, 431 (2004)] is extended here to deal with soluble solutes. This new version that accounts for the mass transfer of surfactant in the bulk phase, as well as for its interfacial adsorption/desorption, is employed in this work to assess the influence of surfactant solubility on the unstable evolution. We confirm previously reported findings: surfactants do not affect the system stability but the growth rate of the instability [D. R. Otis, M. Johnson, T. J. Pedley, and R. D. Kamm, "The role of pulmonary surfactant in airway closure," J. Appl. Physiol. 59, 1323 (1993)] and they do not change the successive shapes adopted by the liquid film as the instability develops [S. Kwak and C. Pozrikidis, "Effects of surfactants on the instability of a liquid thread or annular layer. Part I: Quiescent fluids," Int. J. Multiphase Flow 27, 1 (2001)]. Insoluble surfactants delay the instability process, and the time needed to form liquid lenses disconnecting the gas phase-i.e., the closure time-is four to five times larger than for pure liquids. This retardation effect is considerably reduced when the surfactants are somewhat soluble. For a typical system adopted as a reference case, detailed computed predictions are shown; among them, curves of closure time versus adsorption number are given for solubility values ranging from insoluble to highly soluble conditions. In addition, the evolution of the four mass transport terms appearing in the interfacial mass balance equation-normal and tangential convection, diffusion and sorption - is scrutinized to uncover the mechanisms by which surfactant solubility affects the growth rate of the instability. () 2006 American Institute of Physics. [DOI: 10.1063/1.2173969]
\end{abstract}

\section{INTRODUCTION}

When a viscous fluid contained in a capillary tube is displaced by an immiscible one of smaller viscosity, a residual thin film of the displaced liquid is left along the capillary walls; a usual example is a liquid being displaced by a gas phase. The annular liquid film so formed turns out to be unstable to certain axisymmetric perturbations that, after a while, give rise to the formation of regularly spaced lobes. If the thickness of the deposited film is larger than a certain threshold value, the lobes will grow until they collapse, shaping liquid lenses that leave a disconnected intruding phase.

The phenomena just described have been extensively studied since they constitute appropriate models of events occurring at pore levels for several cases of practical interest. Though the formation and ulterior disruption of a uniform liquid film may occur in a consecutive manner, there is no interaction between these processes because they present characteristic times that are totally different; i.e., the film

\footnotetext{
a) Author to whom correspondence should be addressed. Electronic mail: fasaita@ceride.gov.ar
}

formation is much faster than the film disruption. Therefore, both phenomena have been studied independently of each other.

The analysis of film formation in capillaries was mainly motivated by its connection with transport processes in porous media, in particular with oil recovery processes. Numerous works whose main objective was to predict the thickness of the film deposited on the capillary walls ${ }^{1-9}$ followed the earlier analyses of Fairbrother and Stubb ${ }^{10}$ and Bretherton. ${ }^{11}$

On the other hand, Lord Rayleigh, near the end of the 19th century, initiated studies about the instability of cylindrical thin films. He analyzed two limiting cases without inertia: an inviscid liquid cylinder and a viscous one. ${ }^{12,13}$ Later on, Weber ${ }^{14}$ and Tomotika ${ }^{15}$ considered more realistic cases of liquid threads in unbounded domains. Goren ${ }^{16}$ was the first to study the case of annular liquid films in contact with a solid, i.e., supported on a wire or lining the interior walls of a capillary. He used linear stability analysis to determine the fastest growing mode when either inertia or viscous forces are negligible. Shortly afterwards, significant experimental data were provided by Goldsmith and Mason. ${ }^{17}$ The study of the subject acquired new momentum during the 
last two decades of the 20th century motivated by a disease known as respiratory distress syndrome. This disease has its origin in a deficiency of surfactant that is naturally secreted by the lung tissues; the lack of surfactant favors the instability of the liquid layer lining the lung airways and induces the formation of lenses that restrain the pulmonary function by occluding the air passages.

Theoretical models were developed with the purpose of elucidating the functioning of the lungs; considering the geometry of the airways and the thinnest of the films lining their walls, these models were usually based on lubrication theory that leads to one-dimensional nonlinear evolution equations. ${ }^{18,19}$ They were first employed to determine the critical film thickness, as well as the closure time, i.e., the minimum film thickness needed for the growing unstable lobes to reach the center of the tube and the time needed to do it so, respectively. Gauglitz and Radke ${ }^{19}$ found, for rigid capillary tubes and clean liquids, that the critical film thickness is about 0.12 of the capillary radius.

The one-dimensional models underwent ulterior additions and refinements to allow the examination of other features of lung functioning. Johnson et al. ${ }^{20}$ used an ad hoc methodology in order to consider inertial effects in the onedimensional flow equations; Halpern and Grotberg ${ }^{21}$ examined the influence that wall flexibility has on the process and found it to produce significant changes in the values of critical film thickness. The presence of insoluble surfactants was also considered by Halpern and Grotberg ${ }^{22}$ and Otis et al. ${ }^{23}$ These authors found that surfactants delay the unstable evolution, and the time needed to form the liquid lenses increases four to fives times. These findings were confirmed experimentally by Cassidy et al., ${ }^{24}$ who determined that surfactants can decrease the growth rate on the instability by $80 \%$ and increase the closure time by a factor of 3.8 .

Kwak and Pozrikidis, ${ }^{25}$ who also studied the effects of insoluble surfactants using linear stability analysis, arrived at a similar conclusion; in addition, they used a model based on lubrication theory to follow the instability and found that surfactants do not affect the successive shapes adopted by the liquid film throughout the unstable evolution.

In a work that is the forerunner of the present one, Campana et $a l .{ }^{26}$ studied the Rayleigh instability in a capillary tube with a 2D free surface flow model based on the NavierStokes equations. The objective was to determine the limits of applicability of the lubrication approximation regarding the film relative thickness and the elastic interfacial properties arising from the presence of insoluble surfactants. This model was validated for pure liquids with results of the linear stability theory ${ }^{16}$ and with the experimental results of Goldsmith and Mason. ${ }^{17}$ Then, its predictions were compared with results obtained with models based on lubrication theory-i.e., those represented by one-dimensional nonlinear evolution equations. The comparisons showed that onedimensional models give reasonably good predictions for pure liquids if the film is thin enough; however, as the film becomes either thicker or contaminated by surface-active agents, the predicted closure times are increasingly underestimated.

The objective of the present work is to establish the in- fluence of surfactant solubility on the Rayleigh instability in capillaries; nevertheless, the results for this problem might possibly be extended to other processes governed by interfacial dynamics. For that purpose, an extended version of the 2D free surface flow model already applied by Campana $e t$ $a l .^{26}$ is employed. This new version accounts for mass transfer of solute in the bulk as well as its interfacial adsorption/ desorption, and it is used to evaluate the influence of surfactant solubility on the instability process. This evaluation will be made through changes in closure times since, at least for the values of the parameters here explored, changes in surfactant solubility do not affect the system stability but rather the stability growth rate. According to the results of this model, we must say in advance that the effects produced by surfactants of relatively low solubility appear to be much larger than we expected. ${ }^{26}$

In the following section, the model, the numerical technique employed, and the system selected as a reference case are presented; in Sec. III, the predictions obtained are shown and discussed; and, finally, in Sec. IV a summary of the conclusions is given.

\section{THE MODEL AND THE NUMERICAL TECHNIQUE}

The model adopted considers a cylindrical capillary whose interior walls are lined by a stationary liquid film of uniform thickness containing a small amount of a soluble surfactant. The concentration of surfactant in the bulk, as well as at the interface, is uniform, and there exists equilibrium between them. The liquid is incompressible and Newtonian of density $(\rho)$ and viscosity $(\mu)$; the system is at constant temperature and its dimensions are small enough so that gravity forces might be neglected in comparison with capillary, viscous, and inertial forces. We assume that interfacial viscosity is absent and that the local concentration of surfactant at the interface only changes the local value of surface tension.

We study the time evolution of the system just described by perturbing the horizontal interfacial shape with a sinusoidal wave of small amplitude and a certain wavelength. We mainly focus our attention on how the solubility of the surfactant affects that evolution.

In the first part of this section, our adopted model is translated into its mathematical expression; thus, the governing equations and boundary conditions for the flow and the mass transport problems, followed by the equation of state relating surface tension to surfactant concentration, are presented in this order. In Sec. II B, the numerical technique employed to solve these governing equations is summarized. Finally, in Sec. II C, we adopt the values of the parameters defining a "typical" or "reference case" that will be employed to study the influence of surfactant solubility on this type of capillary-driven instability.

\section{A. Governing equations}

We consider the situation sketched in Fig. 1, where all the variables shown are dimensionless except for the wall radius $(a)$ and the radius of the gas-liquid interface $(b)$. Since (a) has been selected as the characteristic length, $F=(a$ 


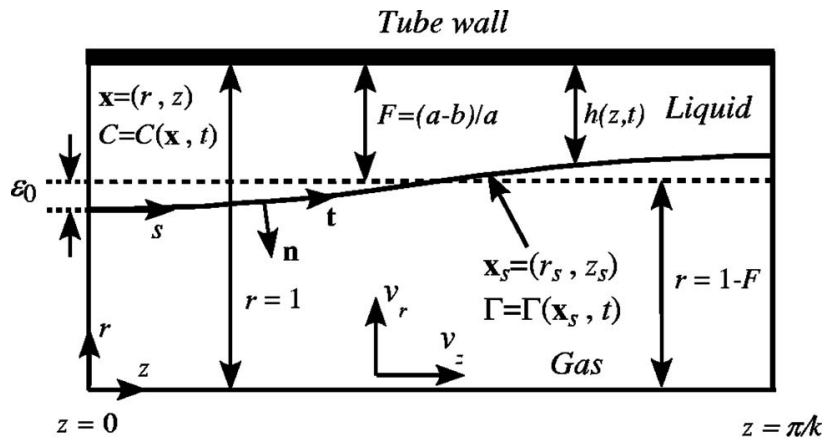

FIG. 1. Simplified illustration of the flow domain and significant dimensionless variables.

$-b) / a$ and $(1-F)$ are the nondimensional initial film thickness and initial radius of the gas-liquid interface, respectively. Figure 1 also depicts the sinusoidal axisymmetric perturbation of wavelength $\lambda$ imposed at first, and the domain of study which just involves half-wavelength since periodic disturbances will be considered only. Therefore, the domain extends from the planes $z=0$ to $z=\pi / k$, where $k=2 \pi a / \lambda$ is the nondimensional wave number of the perturbation. The components of the vector $\mathbf{x}_{S}$-i.e., $\left(r_{S}, z_{S}\right)=\left[1-h\left(z_{S}, t\right), z_{S}\right]$-are the radial and axial coordinates of a point pertaining to the free surface whose location is parametrized by its distance to the tube wall $h\left(z_{S}, t\right)$.

In the next two subdivisions of Sec. II A, we will present the governing equations for both the flow and mass transfer problems, together with their appropriate boundary conditions.

\section{The flow problem}

Since the fluid is considered Newtonian and incompressible of density $\rho$ and constant viscosity $\mu$, the dimensionless mass and momentum balance equations are

$$
\begin{aligned}
& \nabla \cdot \mathbf{v}=0, \\
& \operatorname{Re}\left[\frac{d \mathbf{v}}{d t}+\left(\mathbf{v}-\mathbf{x}^{t}\right) \cdot \nabla \mathbf{v}\right]=\nabla \cdot \mathbf{T},
\end{aligned}
$$

where $\mathbf{T}=-p / \mathrm{Ca} \mathbf{I}+\left(\nabla \mathbf{v}+\nabla \mathbf{v}^{T}\right)$ is the Cauchy stress tensor; $p$ stands for the hydrodynamic pressure, $\mathbf{v}=\left(v_{r}, v_{z}\right)$ for the velocity vector, $t$ for the time, and $\nabla$ is the dimensionless gradient operator in cylindrical coordinates. Equations (1) and (2) have been put into their dimensionless form by using well known appropriate scales for this problem (see Hammond $\left.{ }^{18}\right)$. They are $\left(\sigma_{\text {ref }} F^{3} / \mu\right)$ for the velocity vector, $\left(\sigma_{\text {ref }} / a\right)$ for pressure, and $\left(\sigma_{\mathrm{ref}} F^{3} / a\right)$ for the components of the viscous stress tensor; therefore, the time scale results $\left[a \mu /\left(\sigma_{\text {ref }} F^{3}\right)\right]$. In the above expressions, $\sigma_{\text {ref }}$ is the reference gas-liquid surface tension, which will be defined in Sec. II A 3.

Since the shape of the flow domain changes as the instability evolves, the flow equations are expressed by means of an arbitrary Lagrangian-Eulerian (ALE) description. Thus, $d(.) / d t$ in Eq. (2) denotes the temporal derivative following the coordinates of the computational domain, which move with velocity $\mathbf{x}^{t}$ regarding the fixed reference frame $(r, z)$.
Although the velocity $\mathbf{x}^{t}$ might be arbitrary, several works proved that if $\mathbf{x}^{t}$ is set proportional to the velocity of the interfacial particles - the choice we made in this work-a convenient description of the motion is accomplished. ${ }^{26-28}$

The procedure applied to obtain Eqs. (1) and (2) allows the physicochemical and geometric parameters to be collected in two numbers: (i) a modified Reynolds number $\mathrm{Re}$ $=\rho V a / \mu=\left(\rho a \sigma_{\text {ref }} F^{3} / \mu^{2}\right)$ which relates inertial, viscous, and capillary forces, and (ii) the Capillary number $\mathrm{Ca}=V \mu / \sigma_{\text {ref }}$ which measures the relative magnitude of viscous and capillary forces. According to the characteristic value just adopted for $V$, Ca turns out to be equal to $F^{3}$.

Since the interface location $h\left(z_{S}, t\right)$ is unknown, an additional equation is needed to solve the system given by (1) and (2). That equation is provided by the kinematic condition stating that the normal component of the velocity vector at the interface equals the velocity of the particles lying on it. This is the usual assumption that regards the interface as a material surface:

$$
\left.(\mathbf{n} \cdot \mathbf{v})\right|_{S}=\mathbf{n} \cdot \mathbf{x}_{S}^{t}
$$

The following sinusoidal perturbation of amplitude $\varepsilon_{0}$ and wave number $k$ is imposed on the interface to trigger the instability,

$$
h(z, 0)=F\left[1+\varepsilon_{0} \cos (k z)\right] .
$$

The solution of the system represented by Eqs. (1)-(3) and the periodic perturbation (4) needs appropriate boundary conditions. For that purpose, the usual no-slip conditions are used at the capillary wall $\left(v_{r}=v_{z}=0\right)$, and the so-called symmetry conditions are imposed at the planes $z=0$ and $z=\pi / k$; the latter are expressed as

$$
v_{z}=0 ; \quad \frac{\partial v_{r}}{\partial z}=0 ; \quad \frac{\partial h}{\partial z}=0 .
$$

We consider a Newtonian interface ${ }^{29}$ enclosing an inviscid gas core whose constant uniform pressure - arbitrarily set to zero-is taken as reference. Consequently, the normal component of the stress vector at the interface is balanced by the combined contribution of surface tension and interfacial curvature, while the tangential component can only be equilibrated by the Marangoni stresses arising from the gradients of the interfacial concentration of surfactant. This equilibrium can be stated as

$$
\mathbf{T} \cdot \mathbf{n}=\frac{1}{\mathrm{Ca}}\left[\sigma \kappa \mathbf{n}+\nabla_{S} \sigma\right] .
$$

In Eq. (6) $\kappa$ is the sum of both axial and azimuthal curvatures, $\sigma=\sigma(s)$ is the dimensionless surface tension (scaled with $\sigma_{\text {ref }}$ ) that locally depends on the concentration of surfactant, and $\nabla_{S}$ is the interfacial gradient operator with components in $\theta$ and $s$ directions. The $\theta$ axis coincides with the azimuthal one in the cylindrical frame and is normal to the plane defined by both $z$ and $r$ axes, while $s$ represents the interfacial arc length, which is defined positive in the direction of the tangential vector $\mathbf{t}$ (see Fig. 1). 


\section{The mass transfer problem}

In the bulk phase, convection and diffusion transport the solute; thus, the dimensionless ALE form of the surfactant mass balance equation is given by

$$
\frac{d C}{d t}+\left[\left(\mathbf{v}-\mathbf{x}^{t}\right) \cdot \nabla C\right]-\frac{1}{\mathrm{Pe}} \nabla^{2} C=0,
$$

where the first term on the left gives the time change of the concentration following the motion of the computational coordinates. In Eq. (7), the diffusive flux is described by Fick's law, $\mathrm{Pe}=V a / D=F^{3} \sigma_{\text {ref }} a /(D \mu)$ is the Peclet number in the bulk, $D$ being the diffusion coefficient of the surfactant, which is assumed constant and isotropic. The nondimensional concentration of surfactant $C$ is measured in units of the uniform initial concentration $C_{0}$; thus, the initial condition for Eq. (7) will be $C(\mathbf{x}, t=0)=1$. Since the tube wall is impervious and the planes at $z=0$ and at $z=\pi / k$ are symmetry planes, the appropriate boundary conditions for Eq. (7) are

$(\mathbf{n} \cdot \nabla C)=\frac{\partial C}{\partial r}=0 \quad$ at $r=1, \quad 0 \leqslant z \leqslant \pi / k$,

$(\mathbf{n} \cdot \nabla C)=\frac{\partial C}{\partial z}=0 \quad$ at $z=0, \quad z=\pi / k \quad$ and $\quad r_{S}(t) \leqslant r \leqslant 1$.

The region of the bulk that is just in contact with the interface is named the subsurface; this is a very thin layer where solute is transported by diffusion only, and is transferred to/from the interface by an adsorption/desorption process. If $j_{\mathbf{n}}^{*}$ denotes the dimensional sorptive flux, the mass conservation principle requires this flux to be equal to the diffusive flux in the subsurface; that is,

$$
j_{\mathbf{n}}^{*}=-D\left(\mathbf{n} \cdot \nabla^{*} C^{*}\right) \text {. }
$$

Equation (9) is the boundary condition to be applied at the interface in Eq. (7), and its particular expression will depend on the kinetic of sorption adopted. The asterisk in (9) - and in what follows - signals a dimensional quantity; the only dimensional quantities not marked with asterisks are just physical properties $(\rho, \mu, D$, etc.) or fixed values of the variables such as initial concentrations $\left(C_{0}, \Gamma_{0}\right.$, etc.).

The equation for the mass balance of surfactant at a moving interface can be expressed as ${ }^{29,30}$

$$
\frac{d \Gamma}{d t}-\mathbf{x}_{S}^{t} \cdot \nabla_{S} \Gamma+v_{(\mathbf{n})} \Gamma\left(\nabla_{S} \cdot \mathbf{n}\right)+\nabla_{S} \cdot\left(\mathbf{v}_{S} \Gamma\right)-\frac{1}{\mathrm{Pe}_{S}} \nabla_{S}^{2} \Gamma=j_{\mathbf{n}},
$$

where $\Gamma$ is the nondimensional interfacial concentration measured in terms of the uniform initial one $\left(\Gamma_{0}\right)$; the latter is in equilibrium with the initial bulk concentration $C_{0}$. The $S$ subscripts indicate surface quantities; therefore, $\mathbf{x}_{S}$ represents the moving coordinates of interfacial points and $\mathbf{x}_{S}^{t}$ their velocity with respect to the fixed reference frame $(r, z)$ adopted. Furthermore, $\mathbf{v}_{S}=(\mathbf{v} \cdot \mathbf{t})_{S} \mathbf{t}$ and $v_{(\mathbf{n})}=(\mathbf{v} \cdot \mathbf{n})_{S}$ are the tangential and normal component of the interfacial velocity vector, respectively, while $d \Gamma / d t=(\partial \Gamma / \partial t)_{\mathbf{x}_{S}}$. In Eq. (10) $\mathrm{Pe}_{S}=V a / D_{S}$ $=F^{3} \sigma_{\text {ref }} a /\left(D_{S} \mu\right)$ denotes the interfacial Peclet number and
$D_{S}$ represents the interfacial diffusion coefficient, which is assumed constant and isotropic.

Finally, the initial condition to be used in Eq. (10) is $\Gamma\left(\mathbf{x}_{S}, t=0\right)=1$, and we impose symmetry conditions at the extremes of the interface $\left(s=0\right.$ and $s=s_{f}$ which have the axial coordinates $z=0$ and $z=\pi / k$, respectively) implying that both convective and diffusive mass transport are zero at those locations. These conditions are satisfied when the interfacial velocities, as well as the interfacial concentration gradients, are zero there. Thus, the first condition-which has already been enforced [see Eq. (5)]-is

$\mathbf{t}= \pm \mathbf{e}_{z} \Rightarrow \mathbf{v}_{S}= \pm v_{z} \mathbf{e}_{z}=0 \quad$ at $s=0 \quad$ and $\quad s=s_{f}, \quad \forall \theta$,

while the second one is

$\mathbf{t} \cdot \nabla_{S} \Gamma=\frac{d \Gamma}{d s}=0 \quad$ at $s=0 \quad$ and $\quad s=s_{f}, \quad \forall \theta$.

A possible representation for the sorptive flux at the interface $\left(j_{\mathbf{n}}^{*}\right)$ in Eq. (9) is the use of a Langmuir-type kinetic expression, that is, ${ }^{29}$

$$
j_{\mathbf{n}}^{*}=k_{a} C_{S}^{*}\left(\Gamma_{\infty}-\Gamma^{*}\right)-k_{d} \Gamma^{*} .
$$

In Eq. (13), $\Gamma_{\infty}$ is the concentration of surfactant that saturates the interface, $C_{S}^{*}$ is the dimensional subsurface concentration, while $k_{a}$ and $k_{d}$ are the adsorption and desorption constants, respectively. However, if the interface is very dilute, i.e., $\Gamma^{*} \ll \Gamma_{\infty}$, the kinetic expression (13) can be approximated by its linear version,

$$
j_{\mathbf{n}}^{*}=k_{a} C_{S}^{*} \Gamma_{\infty}-k_{d} \Gamma^{*} .
$$

At equilibrium, the sorptive flux is null; thus, the concentrations of surfactant in the bulk and at the interface are related by the following isotherm:

$$
C_{S}^{*}=\frac{k_{d}}{k_{a}} \frac{\Gamma^{*}}{\Gamma_{\infty}} .
$$

Since the initial state of the system is an equilibrium state, Eq. (15) is also satisfied when the concentrations of surfactant are $C_{0}$ and $\Gamma_{0}$; therefore, the dimensionless version of Eq. (14) becomes

$$
j_{\mathbf{n}}=\alpha\left(C_{S}-\Gamma\right),
$$

where $\alpha=a k_{d} / V=a k_{d} \mu /\left(F^{3} \sigma_{\text {ref }}\right)$ —usually known as "adsorption number" - can be interpreted as the time scale ratio between flow and adsorption. In addition, the nondimensional form of the boundary condition provided by Eq. (9) is

$$
-1 / \operatorname{Pe}(\mathbf{n} \cdot \nabla C)=\alpha K\left(C_{S}-\Gamma\right) .
$$

The dimensionless parameter $K=\Gamma_{0} /\left(a C_{0}\right)$ in Eq. (17) is the so-called solubility number; its value can be thought of as a measure of the surfactant solubility or it can be considered as the ratio of the amounts of surfactant present at the interface and in the bulk phase, respectively. That is, if $K$ is very large, it means that either an almost insoluble surfactant is being considered or the interface contains much more surfactant than the bulk phase-notice that, regardless of the 


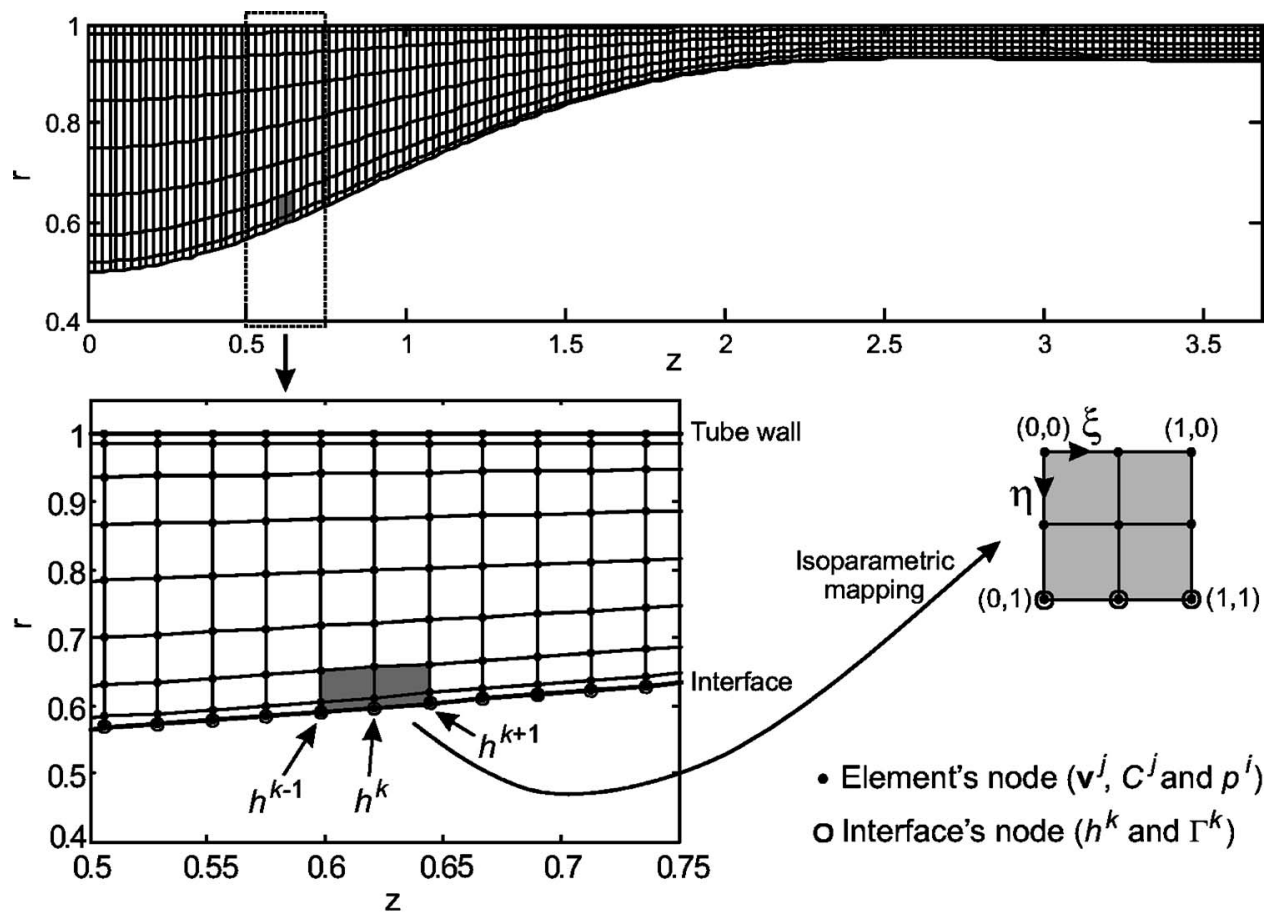

FIG. 2. Typical tessellation used to solve the RC (above) and a detailed view of a free-surface element and its isoparametric mapping (below).

surfactant solubility, the latter case might be true if the system is small enough. The opposite must be interpreted when $K$ is very small.

\section{The surface equation of state}

The model is completed with a surface equation of state; i.e., with an expression that relates the surface tension and the interfacial concentration of surfactant. Since we supposed that $\Gamma^{*} \ll \Gamma_{\infty}$, Gibb's thermodynamics relationship is employed,

$$
d \sigma^{*}=-R_{g} T \Gamma^{*} d\left[\ln C_{S}^{*}\right],
$$

where $R_{g}$ is the universal gas constant and $T$ is the absolute temperature. If a reference state is represented by $\left(\sigma_{\text {ref }} ; \Gamma_{\text {ref }}\right)$, the foregoing equation - with the aid of Eq. (15) — can be integrated to give the simplest version of the Langmuir equation of state,

$$
\sigma^{*}=\sigma_{\text {ref }}-R_{g} T\left(\Gamma^{*}-\Gamma_{\text {ref }}\right) \text {. }
$$

If we choose the initial state of the system as our reference state, i.e., $\Gamma_{\text {ref }}=\Gamma_{0}$ and $\sigma_{\text {ref }}=\sigma_{0}$, the dimensionless version of Eq. (19) becomes

$$
\sigma=1-\beta(\Gamma-1),
$$

where the nondimensional parameter $\beta=R_{g} T \Gamma_{0} / \sigma_{0}$ is the socalled elastic number; $\beta$ can be interpreted as a measure of the surfactant strength.
Equations (1)-(3), (7), (10), and (20), together with their corresponding initial and boundary conditions, constitute the mathematical representation of our model, whose solution will provide the unknown values of $\mathbf{v}(\mathbf{x}, t), p(\mathbf{x}, t), C(\mathbf{x}, t)$, $\Gamma\left(\mathbf{x}_{S}, t\right)$ and the interface location $\left[\mathbf{x}_{S}(t)\right]$ too.

In the next section we briefly describe the most salient features of the numerical technique used to translate this set of equations into an analogous discrete one, as well as the procedures employed to solve it.

\section{B. Numerical technique}

The equation set was solved numerically by the Galerkin-finite-element technique, and the spine method was used to achieve a suitable parametrization of the free surface. Since this procedure is completely standard and it is well described in the literature, ${ }^{27,28,31}$ just a short summary follows.

The flow domain is partitioned by means of quadrilateral elements which are delimited by two vertical straight lines in their lateral sides, i.e., the spines, and by two curved lines (on top and bottom sides) that follow the shape of the free surface (see Fig. 2). Each element contains nine nodes, each having three degrees of freedom (axial and radial components of the velocity vector and the bulk concentration of surfactant) except for the four nodes located at the corners that present an additional degree: the pressure. Mixed interpolation is employed: biquadratic basis functions $(\phi)$ are used for both velocities and concentration and bilinear ones $\psi$ for pressure. Then, the variables are approached at element level by 


$$
\begin{aligned}
& \mathbf{v}(\mathbf{x}, t)=\sum_{j=1}^{9} \mathbf{v}^{j}(t) \phi^{j}(\xi, \eta), \\
& C(\mathbf{x}, t)=\sum_{j=1}^{9} C^{j}(t) \phi^{j}(\xi, \eta), \\
& p(\mathbf{x}, t)=\sum_{i=1}^{4} p^{i}(t) \psi^{i}(\xi, \eta) .
\end{aligned}
$$

The $(\xi, \eta)$ variables in Eq. (21) are the local coordinates defined on the standard unit square, onto which the domain elements are isoparametrically mapped. The coordinates of the domain $(r, z)$ are also interpolated with the biquadratic basis functions,

$$
\begin{aligned}
& z(\xi, \eta)=\sum_{j=1}^{9} z^{j} \phi^{j}(\xi, \eta), \\
& r(\xi, \eta, t)=\sum_{j=1}^{9} r^{j}(t) \phi^{j}(\xi, \eta) .
\end{aligned}
$$

As can be observed in Eq. (22), the axial coordinate of the nodes remains constant while the radial coordinate changes with time to follow the evolution of the flow domain. Figure 2 shows that the free surface can easily be described on the elements pertaining to the interface as a line of constant $\eta(\eta=1)$. Then, if the free surface is parametrized by $h^{k}(t)$ (the distance along the spine between the tube wall and the free surface), it can be interpolated by the one-dimensional specialization (three nodes) of the biquadratic basis functions $\left[\widetilde{\phi}^{k}(\xi)=\phi^{k}(\xi, \eta=1)\right]$ as

$$
r_{S}(\xi, t)=1-\sum_{k=1}^{3} h^{k}(t) \widetilde{\phi}^{k}(\xi) .
$$

The interfacial concentration of surfactant $\Gamma(\xi, t)$ is also interpolated with the aid of the one-dimensional quadratic basis functions,

$$
\Gamma(\xi, t)=\sum_{k=1}^{3} \Gamma^{k}(t) \widetilde{\phi}^{k}(\xi)
$$

To account for these unknowns $\left[h^{k}(t)\right.$ and $\left.\Gamma^{k}(t)\right]$, two additional degrees of freedom are assigned to the interfacial nodes, and the weighted residuals of both the kinematics and interfacial mass balance equations are employed to obtain the coefficients of the expansions (23) and (24), respectively. The nodes of the domain move along the spines in a previously defined manner: the nodal position over the spine is proportional to the interfacial height associated with this spine. Therefore, the unknowns $h^{k}(t)$ are calculated simultaneously with the remaining variables (velocity, pressure, and concentrations), and the coordinates of the nodes and the shape of the domain are updated fitting in the interfacial evolution.

Owing to the axial symmetry, the problem is solved in a 2D domain using the Galerkin approach. To this end, the governing equations, weighted with the same interpolating functions used to expand the solution, are integrated in their corresponding domain. As a result, the vanishing residuals are obtained at each node. This technique is well known and it has been widely applied in free surface flow problems (see Khesghi and Scriven ${ }^{27}$ ). Since the novel aspect of this work is related to the mass transfer of surfactant, we will provide details of the residual forms of Eqs. (7) and (10) exclusively.

The procedure explained above yields the following vanishing residuals of the mass balance in the bulk phase and at the interface, respectively:

$$
R_{C B}^{j}=\int_{\Omega} \phi^{j}\left\{\left[C^{t}+\left(\mathbf{v}-\mathbf{x}^{t}\right) \cdot \nabla C\right]-1 / \operatorname{Pe}(\nabla \cdot \nabla C)\right\} d \Omega=0,
$$

$$
\begin{aligned}
R_{C S}^{k}= & \int_{S} \widetilde{\phi}^{k}\left\{\Gamma^{t}-\mathbf{x}_{S}^{t} \cdot \nabla_{S} \Gamma+\Gamma v_{(\mathbf{n})}\left(\nabla_{S} \cdot \mathbf{n}\right)-\alpha\left(C_{S}-\Gamma\right)\right. \\
& \left.+\nabla_{S} \cdot\left[\left(\Gamma \mathbf{v}_{S}\right)-1 / \mathrm{Pe}_{S} \nabla_{S} \Gamma\right]\right\} d S=0 .
\end{aligned}
$$

In the foregoing $d \Omega=r d r d z$ and $d S=r_{S} d s$; then, if the divergence theorem is applied to the last term in both equations, the result is

$$
\begin{aligned}
R_{C B}^{j}= & \int_{\Omega} \phi^{j}\left\{\left[C^{t}+\left(\mathbf{v}-\mathbf{x}^{t}\right) \cdot \nabla C\right]+1 / \operatorname{Pe}\left(\nabla \phi^{j} \cdot \nabla C\right)\right\} r d r d z \\
& +\int_{S} \phi^{i}\{1 / \operatorname{Pe}(-\mathbf{n} \cdot \nabla C)\} r_{S} d s=0 \\
R_{C S}^{k}= & \int_{S}\left\{\widetilde{\phi}^{k}\left[\Gamma^{t}-\mathbf{x}_{S}^{t} \cdot \nabla_{S} \Gamma\right]+\widetilde{\phi}^{k} \Gamma v_{(\mathbf{n})}\left(\nabla_{S} \cdot \mathbf{n}\right)\right. \\
& -\nabla_{S} \widetilde{\phi}^{k} \cdot\left(\Gamma \mathbf{v}_{S}\right)+1 / P e_{S}\left(\nabla_{S} \widetilde{\phi}^{k} \cdot \nabla_{S} \Gamma\right) \\
& \left.-\widetilde{\phi}^{k} \alpha\left(C_{S}-\Gamma\right)\right\} r_{S} d s+\left\{\widetilde { \phi } ^ { k } \left[\Gamma\left(\mathbf{v}_{S} \cdot \mathbf{t}\right)\right.\right. \\
& \left.\left.\left.-1 / \operatorname{Pe}_{S}\left(\nabla_{S} \Gamma \cdot \mathbf{t}\right)\right] r_{S}\right\}\right\}_{s=0}^{s=s_{f}}=0 .
\end{aligned}
$$

The line integral appearing in Eq. (27) represents the diffusive mass flux at the domain boundaries. At the free surface, the integrand between brackets is conveniently substituted by condition (17) while at the remaining boundaries (symmetry planes and tube wall) it is eliminated to enforce-in a weak mode-symmetry and impermeability [see Eq. (8)].

The last term shown in Eq. (28) accounts for the added contribution of convective and diffusive mass transport at both ends of the interfacial domain; according to the symmetry conditions imposed there [see Eqs. (5), (11), and (12)], that term is eliminated. Considering that in axial symmetry the surface gradient operator usually simplifies to $d(.) / d s \mathbf{t}$ and $\nabla_{S} \cdot \mathbf{n}=-\left(\kappa_{1}+\kappa_{2}\right)$, being $\kappa_{1}$ and $\kappa_{2}$ the axial and azimuthal curvatures, respectively, Eq. (28) can be rewritten as 


$$
\begin{aligned}
R_{C S}^{k}= & \int_{S}\left\{\widetilde{\phi}^{k}\left[\Gamma^{t}-\mathbf{x}_{S}^{t} \cdot \frac{d \Gamma}{d s} \mathbf{t}\right]-\widetilde{\phi}^{k} \Gamma v_{(\mathbf{n})}\left(\kappa_{1}+\kappa_{2}\right)\right. \\
& -\frac{d \widetilde{\phi}^{k}}{d s} \mathbf{t} \cdot\left(\Gamma \mathbf{v}_{S}\right)+1 / \mathrm{Pe}_{S}\left(\frac{d \widetilde{\phi}^{k}}{d s} \mathbf{t} \cdot \frac{d \Gamma}{d s} \mathbf{t}\right) \\
& \left.-\widetilde{\phi}^{k} \alpha\left(C_{S}-\Gamma\right)\right\} r_{S} d s=0 .
\end{aligned}
$$

Since the interface is represented by a line where $\eta$ is constant, the normal and tangential unit vectors to the free surface can be readily computed from the isoparametric mapping $;{ }^{27}$ then, the curvatures are obtained directly by $\kappa_{1}$ $=d \mathbf{t} / d s \cdot \mathbf{n}$ and $\kappa_{2}=-\left(\mathbf{n} \cdot \mathbf{e}_{r}\right) / r_{S}$.

The spatial tessellation and the Galerkin-finite-element technique yield a set of ordinary differential equations in the discrete variables $\mathbf{v}^{j}(t), C^{j}(t), p^{i}(t), h^{k}(t)$, and $\Gamma^{k}(t)$. This system was integrated in time with a second-order predictorcorrector scheme and for that purpose we choose the AdamsBashforth formula and the trapezoidal rule, respectively. Then, at each time step the algebraic nonlinear system was solved by Newton's method; the iterative process was finished when the norm of the difference between two consecutive vector approximations was $10^{-6}$ or smaller. The time step was adaptively adjusted with the aid of a heuristic method proposed by Crisfield ${ }^{32}$ that attempts to solve Newton's loop in a constant number of iterations. To this end, by using constant coefficients, the method adapts the size of the step if the previous convergence was attained in a number of iterations different from the target sought. This very simple scheme proved to be more convenient than the use of more sophisticated procedures. ${ }^{33,34}$

The computational code was programmed in FORTRAN and it uses the PETSc routines ${ }^{35}$ to solve the linear system of equations. The size of the problem was variable and the number of elements in both axial (NEZ) and radial directions $(N E R)$ was changed considering the domain aspect ratio. For example, three meshes were used to solve the case that we will define as standard in the next section, $N E Z \times N E R=40$ $\times 2$ (1500 degrees of freedom), $N E Z \times N E R=80 \times 4$ (5074 degrees of freedom), and $N E Z \times N E R=160 \times 8$ (18462 degrees of freedom). The three meshes predict similar values of closure time with a deviation below 1\%; however, only the cases of $80 \times 4$ and $160 \times 8$ show a good flowfield description. Therefore, we adopted the mesh of $80 \times 4$ elements, which was appropriate for all simulations since $F$ and $k$ were kept constant throughout this work.

Figure 2 shows a nonuniform distribution of elements in the radial direction; they are concentrated near the interface and also near the wall; this technique was applied to better describe the gradients of velocities and concentrations in those places. Although we could have used a more sophisticated method to produce local refinement (cf. Severino et $a l^{7}$ ), we found this unnecessary because the variables and their derivatives were accurately calculated with the tessellation shown in Fig. 2.

Finally, we must remark that the total mass conservation
TABLE I. Relevant physical magnitudes: Usual range of values and those chosen for our reference case.

\begin{tabular}{ccc}
\hline \hline Physical magnitude & Range of values & Selected value \\
\hline$\Gamma_{\infty}\left[\mathrm{mol} / \mathrm{m}^{2}\right]$ & $10^{-6}-10^{-5}$ & $5 \times 10^{-6}$ \\
$C_{0}\left[\mathrm{~mol} / \mathrm{m}^{3}\right]$ & $10^{-2}-200$ & $5 \times 10^{-2}$ \\
$k_{a}\left[\mathrm{~m}^{3} / \mathrm{mol} \mathrm{s}\right]$ & $0.2-400$ & 50 \\
$k_{d}[1 / \mathrm{s}]$ & $50-180$ & 50 \\
$\Gamma_{0}=\Gamma_{\infty} k_{a} C_{0} / k_{d}\left[\mathrm{~mol} / \mathrm{m}^{2}\right]$ & - & $2.5 \times 10^{-7}$ \\
$D\left[\mathrm{~m}^{2} / \mathrm{s}\right]$ & $10^{-10}-10^{-9}$ & $10^{-9}$ \\
$D_{S}\left[\mathrm{~m}^{2} / \mathrm{s}\right]$ & $10^{-10}-10^{-8}$ & $10^{-9}$ \\
$\sigma_{r e f}[\mathrm{~N} / \mathrm{m}]$ & {$[15-75] \times 10^{-3}$} & $40 \times 10^{-3}$ \\
$\mu[\mathrm{Pa} \mathrm{s}]$ & $10^{-3}-0.1$ & $10^{-2}$ \\
$a[\mathrm{~m}]$ & $10^{-4}-10^{-3}$ & $5 \times 10^{-4}$ \\
$\rho\left[\mathrm{Kg} / \mathrm{m}^{3}\right]$ & $800-1400$ & 1100 \\
\hline \hline
\end{tabular}

of surfactant (bulk+interface) was always verified and the results were accepted provided the errors were smaller than $0.1 \%$.

\section{The reference case}

In order to concentrate our analysis on the surfactant solubility and their effects on the Rayleigh instability, we must adopt appropriate values for the dimensionless parameters defined in Sec. II A. For that purpose, in Table I we summarize the range of values usually found for the physical magnitudes involved; they were taken from several sources, among them we remark the work of Chang and Franses ${ }^{36}$ that gives an extensive characterization of soluble surfactants. These authors studied surfactants whose values of $\mathrm{Ka}$ range from $10^{-10}$ to $10^{-3} \mathrm{~m}$ for highly and weakly soluble, respectively. Therefore, when we refer to a somewhat soluble surfactant we mean values of $K a$ slightly smaller than $10^{-3} \mathrm{~m}\left(\right.$ e.g., $\left.10^{-5} \mathrm{~m}\right)$.

We also want to determine for Rayleigh instability the conditions under which the usual assumption of insolubility is appropriate. Having this purpose in mind, we have chosen the values depicted in the last column of Table I that might represent a system with average physical properties and a somewhat soluble surfactant.

Otis et $_{\mathrm{al}}{ }^{23}$ found that surfactants do not affect the volume of liquid necessary for bridge formation (i.e., the critical volume) and Kwak and Pozrikidis ${ }^{25}$ found that they do not affect the evolution path followed by the unstable system. On this basis, we presumed that for the present problem film thickness is not as relevant as other variables like $K$ or $\alpha$; thus, we fixed $F$ at 0.18 , which is convenient for our purposes. Additionally, for $\varepsilon_{0}$-i.e., the amplitude of the sinusoidal perturbation-we adopted $10^{-3}$.

We will employ the dimensionless wave number corresponding to the most dangerous unstable mode $\left(k^{\max }\right)$ for $F$ $=0.18$ and pure liquids, as predicted by linear stability analysis $\left(\right.$ Goren $\left.^{16}\right)$. Though is known that the value of $k^{\max }$ depends on elastic effects, this dependency appears to be very weak. Actually, we compared the values calculated for pure liquids with those calculated for liquids containing an in- 
TABLE II. Predictions of $k^{\max }$ : by Goren $(G)$ for pure liquids, and by Kwak and Pozrikidis $(K P)$ and the present model, for a liquid contaminated with insoluble surfactant $\left(\beta=0.1, \mathrm{Pe}_{S}=10^{4}\right)$.

\begin{tabular}{cccc}
\hline \hline$F$ & $G$ & $K P$ & Present work \\
\hline 0.1 & 0.776 & 0.777 & 0.780 \\
0.3 & 0.957 & 0.957 & 0.950 \\
0.5 & 1.204 & 1.120 & 1.110 \\
0.7 & 1.448 & 1.433 & 1.430 \\
\hline \hline
\end{tabular}

soluble surfactant with $\beta=0.1$; the comparison was made for several values of film thickness and the results are summarized in Table II.

The second column of Table II depicts the values of $k^{\max }$ for pure liquids as predicted by the linear stability analysis of Goren. The third and fourth columns depict the values of $k^{\text {max }}$ for a system with an insoluble surfactant as predicted by the linear stability analysis of Kwak and Pozrikidis, ${ }^{25}$ and the predictions obtained from our code, respectively. Two features must be remarked from these results: one is the small discrepancies between the values of the third and fourth columns (errors are below 1\%) indicating that our results agree well with those of linear stability analysis. The second one is the small influence the surfactants have on the values of $k^{\max }$ when the film thickness is not larger than 0.3. This characteristic supports our choice of $k^{\text {max }}$ taking in account that we are going to consider soluble surfactants with $\beta \leqslant 0.1$

Finally, we assume isothermal condition with $T=300 \mathrm{~K}$; then $R_{g} T=2493 \mathrm{~m}^{3} \mathrm{~Pa} / \mathrm{mol}$, and Table III summarizes the values of the dimensionless parameters that define our reference case ( $\mathrm{RC})$.

The results of the next section refer to the $\mathrm{RC}$ with an elastic number $(\beta)$ somewhat larger than the value shown in Table III $(\beta=0.1)$; this choice was just made to make it more evident how the surfactant solubility affects some variables. However, we will see that similar trends are found when the elastic number is 0.01 .

\section{RESULTS AND DISCUSSIONS}

The results here presented pertain to the reference case (RC) whose characteristics have been summarized in Table III, or to a system derived from it by varying either $K$ or $\alpha$-or both simultaneously-in the range $[0-\infty)$.

TABLE III. Values of the dimensionless parameters of the model.

$\begin{array}{cc}F=(a-b) / a & 0.18 \\ \operatorname{Re}=\rho \sigma_{\text {ref }} a F^{3} / \mu^{2} & 1.28 \approx 1 \\ \mathrm{Ca}=F^{3} & 5.8 \times 10^{-3} \\ \alpha=a k_{d} \mu /\left(\sigma_{\text {ref }} F^{3}\right) & 1.07 \approx 1 \\ K=\Gamma_{\mathrm{o}} /\left(C_{o} a\right) & 5 \times 10^{-3} \approx 10^{-2} \\ \mathrm{Pe}=\sigma_{\text {ref }} a F^{3} /(\mu D) & 11664 \approx 10^{4} \\ \mathrm{Pe}_{S}=\sigma_{\text {ref }} a F^{3} /\left(\mu D_{S}\right) & 11664 \approx 10^{4} \\ \beta=R_{g} T \Gamma_{0} / \sigma_{\text {ref }} & 0.015 \\ k^{\max }=2 \pi a / \lambda & 0.8537\end{array}$

\section{A. Free surface shapes}

The first finding that must be remarked is related to the successive interfacial shapes adopted by the system as the instability develops; our results indicate that this evolution is quite independent of the presence of surfactants. That is, surfactants delay the instability process to a certain degree depending upon their intrinsic properties like solubility, elasticity, etc., but they do not change the geometrical configurations adopted by the liquid domain as the instability process evolves. This behavior is exemplified by comparing the interfacial shapes produced by a certain system when the action of the surfactant being present becomes maximum and null, respectively. For that purpose we have selected two cases: (i) the RC of Table III in the limit of $K \rightarrow \infty$ (i.e., an insoluble surfactant) in one instance, and (ii) the RC when $K \rightarrow 0$ and $\alpha \rightarrow \infty$ in the second instance. In order to solve the RC for an insoluble surfactant (i), Eq. (7) and their boundary conditions are ignored and the parameter $\alpha$ is set equal to zero.

The value of $K$ approaching zero characterizes a totally soluble surfactant; i.e., the amount of solute available in the bulk phase is enormous and its concentration cannot be altered by adsorption/desorption; thus, the original equilibrium concentration in the bulk phase can never be altered and $C$ $=C_{S}=1$. This particular case will be identified as CCB, denoting Constant Concentration in the Bulk phase. In addition, $\alpha \rightarrow \infty$ indicates an infinitely fast sorption process; consequently, the interfacial concentration of surfactant at any interfacial location $(s)$ must always be in equilibrium with the bulk concentration in the subsurface $\left(C_{S}\right)$, that is, $\Gamma=1$. Since the interfacial concentration gradient is zero, the system behaves as a pure liquid without elastic effects $[\sigma=1$, see Eqs. (22) and (23)] and the mass transport and flow equations become uncoupled. Therefore, it is straightforward to obtain solutions for case (ii) by setting $\beta=0$ and solving (1) and (2), while the mass transport of solute is ignored.

The cases just described, i.e., (i) insoluble surfactant and (ii) no elastic effects, are the two extreme situations in which the unstable process of the reference case is completed in the longest and shortest periods of time, respectively. Thus, if we show that in these two extreme situations the systems adopt similar configurations as the instability process evolves, we should expect that intermediate situations provided by soluble surfactants would behave similarly.

Figures 3 and 4 show the free surface shapes for the RC with $K \rightarrow \infty$ and with $\beta=0$, respectively; in the first case the instability process is completed at $t_{C}=178.93$ while in the second case the closure time $\left(t_{C}\right)$ is 43.56 (notice that the maximum times registered in both figures are barely smaller than $t_{C}$ since closure has not been completed yet). In addition to the initial free surface location, both figures depict interfacial shapes for four instants of time. Those configurations were selected so that interfacial locations at $z=0$ do not differ by more than $0.5 \%$ for curves labeled with the same number. Then, the mean square error (mse) between corresponding curves was evaluated by 


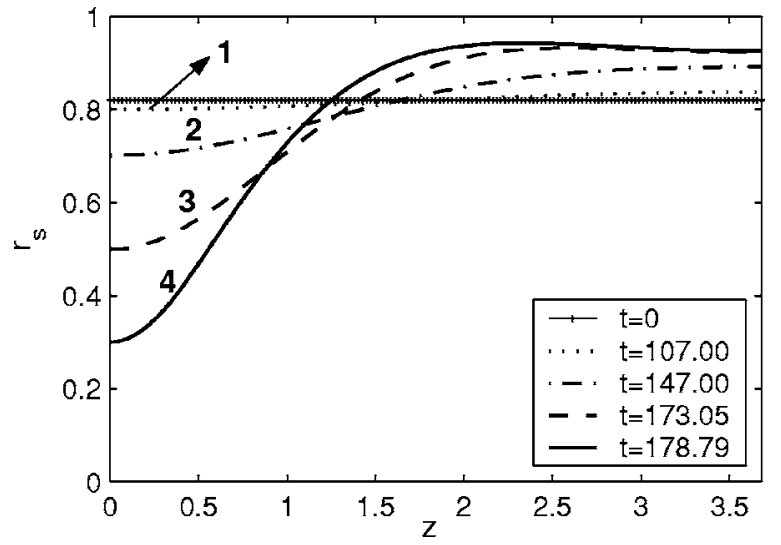

FIG. 3. Free surface shapes for the RC of Table III $(\beta=0.1)$ in the limit of a totally insoluble surfactant $(K \rightarrow \infty)$.

$$
\text { mse }=\left\{\sum_{i=1}^{n} \frac{\left[\Delta r_{s}(i)\right]^{2}}{n}\right\}^{1 / 2}, \quad i=1, \ldots, n .
$$

In the foregoing, $\Delta r_{S}(i)$ is the free surface distance between corresponding curves at node $(i)$ and $n$ is the total number of interfacial nodes. The mean square errors obtained are summarized in Table IV and their small values confirm what is evident from Figs. 3 and 4: the two systems follow the same spatial evolution. Thus, in order to analyze how the Rayleigh instability is affected by the solubility of surfactants, we will comparatively examine several interfacial variables (e.g., velocity components, shear stresses, solute concentrations, etc.) for various systems. For that purpose, comparisons will be made when the systems present similar film thickness at $z=0$.

\section{B. Surfactant solubility and closure times}

We have pointed out before that closure time (the time required for a thick enough film to complete the instability process bridging the interior walls of a capillary) is a crucial parameter in certain processes whose central mechanism is based on Rayleigh instability. However, our main interest here is not focused on the conditions that might promote or

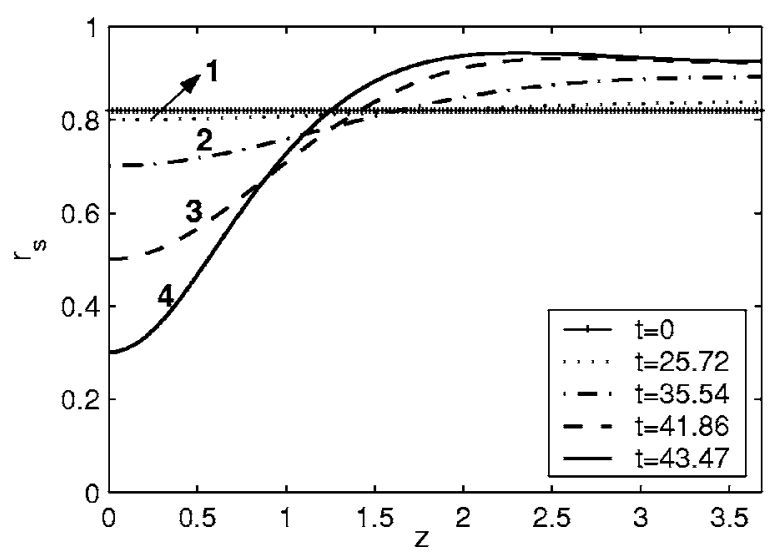

FIG. 4. Free surface shapes for the RC of Table III $(\beta=0.1)$ in the limit of a totally soluble surfactant $(K \rightarrow 0, \alpha \rightarrow \infty)$.
TABLE IV. Mean square error [see Eq. (30)] between the interfacial profiles (1-4) shown in Fig. 3 and the corresponding ones depicted in Fig. 4.

\begin{tabular}{cc}
\hline \hline Instant & mse \\
\hline 1 & $2.97 \mathrm{E}-04$ \\
2 & $1.95 \mathrm{E}-04$ \\
3 & $6.84 \mathrm{E}-04$ \\
4 & $6.92 \mathrm{E}-04$ \\
\hline \hline
\end{tabular}

prevent the formation of liquid lenses, but to use closure times as an indicator of how much the surfactant solubility affects the unstable evolution.

In this section, we will examine the closure time variations when the surfactant considered for the RC is gradually changed from insoluble to completely soluble. Also, the influence of the rate of sorption will be analyzed.

Figure 5 shows our numerical predictions of closure time $\left(t_{C}\right)$ versus the rate of sorption $(\alpha)$ for the four basic solubility values on which we will center our attention; the remaining parameters are those of the RC (see Table III). It is evident that closure times strongly depend on surfactant solubility: while the system with an insoluble surfactant $(K$ $\rightarrow \infty$ ) presents a maximum closure time of 178.93 , the case of no elastic effects-which is the limit of a highly soluble surfactant with an infinite sorption speed $(K \rightarrow 0, \alpha \rightarrow \infty)$-is the other extreme with a closure time of 43.56. Between these extremes, two additional curves are portrayed: one refers to the case of a completely soluble surfactant, and the other refers to a slightly soluble surfactant with $K=0.01$. In these two cases, the closure time varies as the rate of sorption changes; in particular, when the RC is considered (i.e., $\alpha$ $=1$ ), the values are 92.19 and 143.23 , respectively.

In Fig. 6, the curves for $K$ equal to 10, 0.1, and 0.001 are added to those just presented in Fig. 5. From them we might draw the following conclusions:

(1) When $K \geqslant 10$, the solubility is too small and the system behaves as if the surfactants were insoluble. In this case, the closure time does not depend on the value of $\alpha$.

(2) For each value of $K$ there exists an upper bound for $\alpha$ above which the closure time no longer depends on the

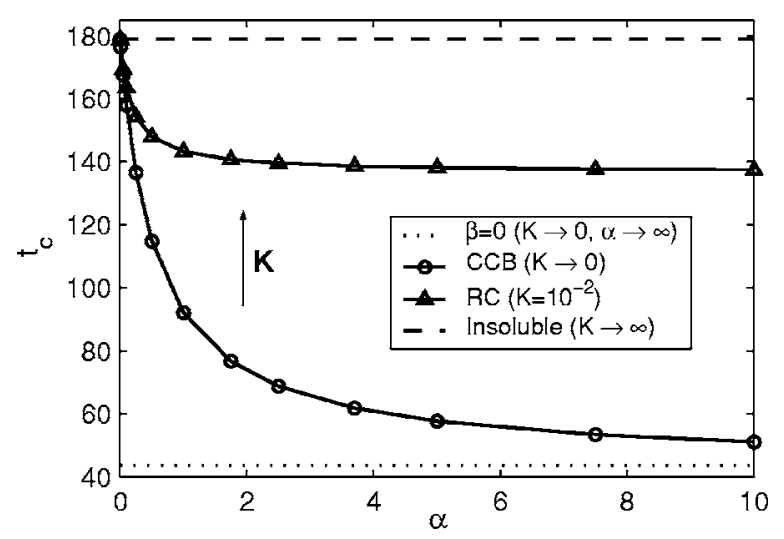

FIG. 5. Closure time vs rate of sorption for the RC of Table III $(\beta=0.1)$ and four conditions of surfactant solubility. 


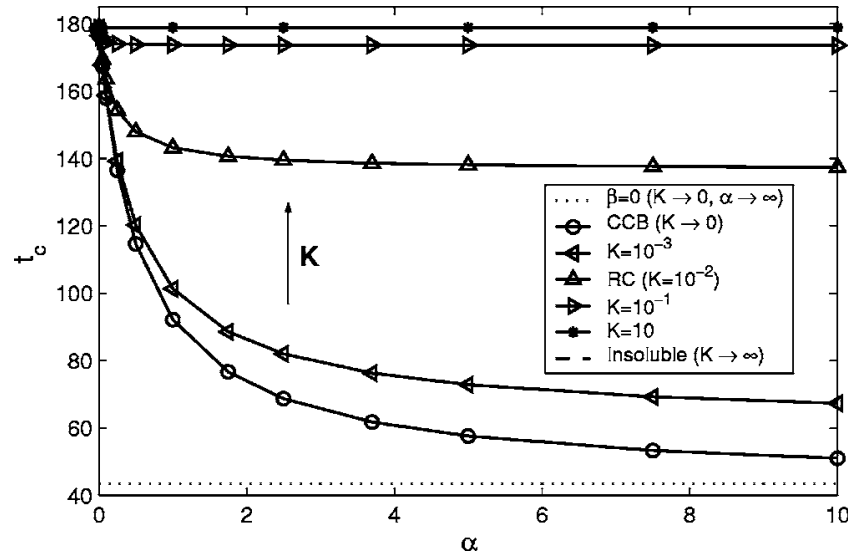

FIG. 6. Predictions shown in Fig. 5 together with the curves of $K=10, K$ $=10^{-1}$, and $K=10^{-3}$.

rate of sorption and $t_{C}$ becomes constant (see Fig. 6).

(3) The upper bound just mentioned increases as the value of $K$ decreases, i.e., as the surfactant becomes more soluble. For the extreme case of CCB the closure time is affected by any value of $\alpha$ in the whole range $[0-\infty)$.

(4) Surfactant solubility might induce considerable changes in closure times; this is the most noticeable feature depicted in Fig. 5. According to the values shown in Tables I and III, a system with $K=0.01$ typifies a somewhat soluble surfactant; nonetheless, a closure time of 143.23 is about $20 \%$ smaller than the closure time produced when the same surfactant is considered insoluble (178.93). More significant is the reduction in the closure time delay (the retardation effect), which is $26.3 \%$ smaller than the closure time delay produced by the insoluble surfactant: (178.93-43.56).

Closure times become constant, as we mentioned in the second conclusion, when at any instant and all along the free surface, the interfacial concentration of surfactant is in equilibrium with the concentration of solute at the subsurface. According to the manner in which we defined the dimensionless concentration, equilibrium occurs when $\left(\Gamma-C_{S}\right) \rightarrow 0$. If the value of $K$ is large, this condition is easily attained with relatively slow sorptions; but, as more soluble surfactants are considered (smaller $K$ ), faster kinetics are needed. This fact might be better understood if one thinks of $K$ as the ratio between the amount of solute present at the interface and that present in the bulk phase. Recalling that $K=\Gamma_{0} /\left(C_{0} a\right)$, we might increase $K$ by reducing the size $a$, signifying that the amount of solute in the bulk has been comparatively reduced. Therefore, smaller amounts of surfactant must be transferred to achieve equilibrium, and this can be done for rather slow sorption kinetics (small values of $\alpha$ ). On the other hand, for CCB $(K \rightarrow 0)$, the amount of surfactant in the bulk is so large that equilibrium can only be reached if $\alpha \rightarrow \infty$.

Figure 7 shows the profiles of $\left(\Gamma-C_{S}\right)$ along the free surface and how they change when the parameter $\alpha$ is increased. If we consider that phase-interface equilibrium is reached when $\left(\Gamma-C_{S}\right) \leqslant\left|10^{-3}\right|$ everywhere along the interface, this condition is accomplished when $\alpha \geqslant 1$ for $K=0.1$,
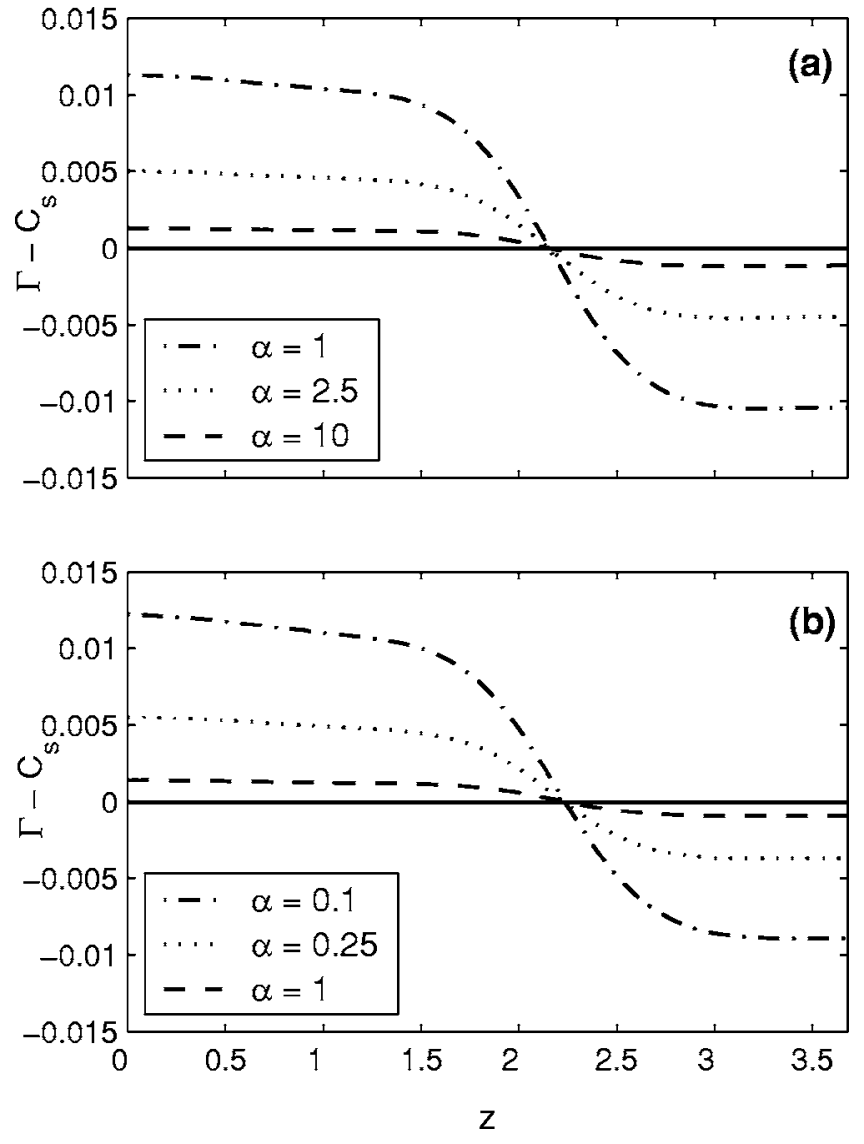

FIG. 7. Phase-interface concentration jump along the free surface: (a) $K$ $=0.01$ and (b) $K=0.1$.

while a value of $\alpha \geqslant 10$ is needed for $K=0.01$. Beyond those values, sorption has no influence on closure times as we mentioned in the second conclusion. In all the cases here portrayed, the evolution of the instability has reached the point where the film height is 0.5 at $z=0$.

Figure 8 portrays the equilibrium concentration profiles along the interface (i.e., the curves of $\Gamma=C_{S}$ ), and how they change with $K$ for values of $\alpha$ large enough. We see that these curves approach the horizontal line of $\Gamma=1$ as $K$ becomes smaller. Also, they indicate that the gradients of $\Gamma$ along the interface $(d \Gamma / d s)$-and the interfacial elastic ef-

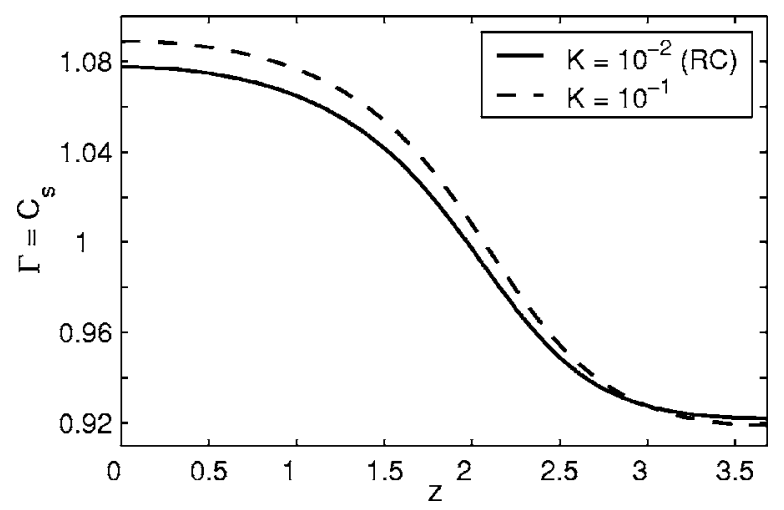

FIG. 8. Interfacial concentration when there is phase-interface equilibrium all along the free surface ( $\alpha$ large enough). Effect of surfactant solubility. 


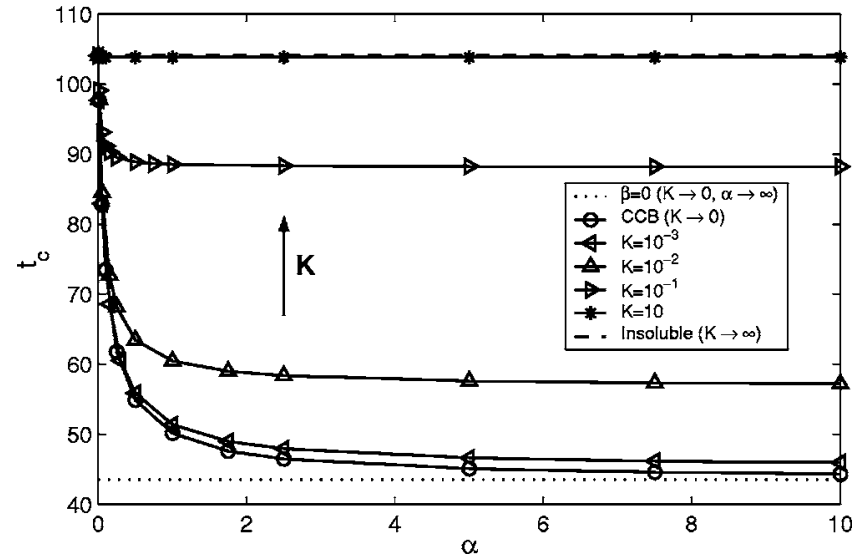

FIG. 9. Closure time vs rate of sorption for the RC of Table III and $\beta$ $=0.01$.

fects as a consequence-decrease as $K$ decreases. As we pointed out before, in the limit of $K \rightarrow 0$ and $\alpha \rightarrow \infty$ the elastic effects become zero since $\Gamma=1$ all along the interface.

The fourth conclusion is remarkable because one expects that surfactants of relatively low solubility might induce smaller changes in the growth rate of the instability. Those changes can even be larger, as Fig. 9 shows for $\beta=0.01$. Under this condition, the closure time of the RC $(K=0.01$, $\alpha=1$ ) is about 61 , while $t_{C}$ is close to 104 when the surfactant is considered insoluble. This signifies a retardation of about 17.5 units of time $(61-43.56)$, which is only $29.0 \%$ of the retardation produced by the insoluble case.

Having shown how the solubility of surfactants affects closure times, in the next section we will try to uncover the underlying mechanisms that produce the behavior just described. For that purpose, we will analyze a limited number of computed predictions and we will examine variables such as velocities, solute concentrations, shear stresses, and different components of the interfacial mass balance.

\section{Surfactant solubility and mechanisms of Rayleigh instability}

In this section, we will concentrate on three systems; they are represented by the reference case with three different solubility conditions, namely the insoluble surfactant case $(K \rightarrow \infty)$, the slightly soluble case $(K=0.01)$, and the totally soluble case $(K \rightarrow 0)$. Their closure times were already presented in Fig. 5 and their values for $\alpha=1$ are 178.93, 143.23, and 92.19, respectively. For the three systems we will make observations at similar stages of the instability, i.e., as we have mentioned before, for similar interfacial configurations. Those configurations are five and they are portrayed in Fig. 10; the curves are labeled from (a) to (e) for increasing times whose values depend on the case we are considering. Table $\mathrm{V}$ summarizes those dimensionless times for all three cases.

The results to be presented will show, for each of the aforementioned systems, how certain interfacial variables change as the instability progresses. Also, with the purpose of exposing the mechanism by which the soluble surfactants

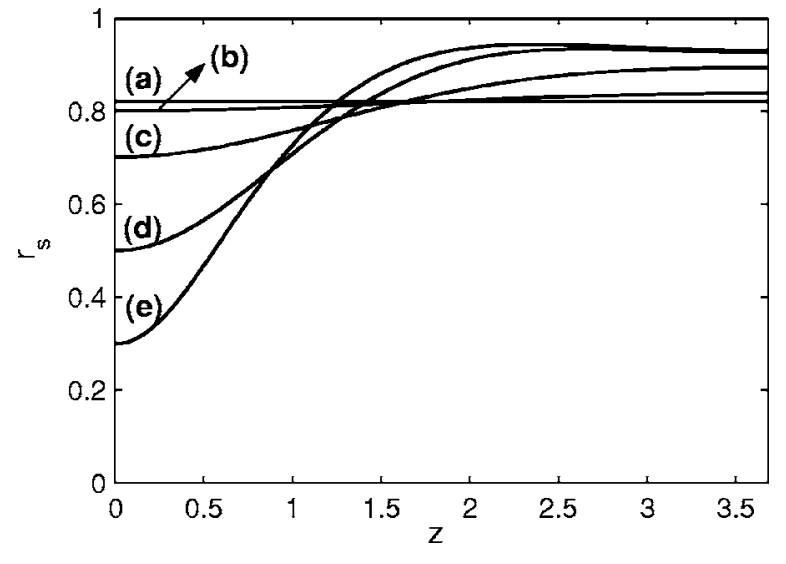

FIG. 10. Interfacial profiles of the five stages used to analyze the influence of surfactant solubility.

delay the instability process, we will show the evolution of the five components of the interfacial mass balance [Eq. (10)].

If Eq. (16) is introduced into Eq. (10), and the latter is rearranged as follows:

$$
\begin{aligned}
d \Gamma / d t-\mathbf{x}_{S}^{t} \cdot \nabla_{s} \Gamma= & -\nabla_{s} \cdot\left(\mathbf{v}_{s} \Gamma\right)-v_{(\mathbf{n})} \Gamma\left(\nabla_{s} \cdot \mathbf{n}\right)+\frac{1}{\operatorname{Pe}_{s}} \nabla_{s}^{2} \Gamma \\
& +\alpha\left(C_{s}-\Gamma\right)
\end{aligned}
$$

it is evident that the local time variation of the interfacial concentration of surfactant - the term on the left side of Eq. (31) — is balanced by the four transport terms appearing on the right side. The first two terms on the right represent the transport by tangential and normal convection, respectively; the third term represents the interfacial diffusion while the fourth takes into account the amount of solute entering/ exiting the interface by adsorption/desorption.

Figures 11(a) and 11(b) show the results for $K \rightarrow \infty$, i.e., for the five stages appearing in the first column of Table $\mathrm{V}$, while Figs. 12(a) and 12(b) and Figs. 13(a) and 13(b) show the results for $K=0.01$ and $K \rightarrow 0$, respectively. We remark that in Figs. 11-13 the results are always labeled as (i), where i stands for a, b, c, d, or e, pointing out that they pertain to the evolution stage labeled in the same way in Fig. 10.

Figure 11(a) depicts the profiles along the free surface of the following variables: interfacial concentration of surfactant, interfacial tangential stresses, and interfacial tangential

TABLE V. Dimensionless time pertaining to the five free-surface profiles shown in Fig. 10 and the closure times, for the three solubility conditions analyzed.

\begin{tabular}{cccc}
\hline \hline Interfacial shape & $K \rightarrow \infty$ & $K=0.01$ & $K \rightarrow 0$ \\
\hline$($ a) & 0 & 0 & 0 \\
$(\mathbf{b})$ & 107.00 & 85.00 & 56.00 \\
$(\mathbf{c})$ & 147.00 & 117.76 & 76.62 \\
$(\mathbf{d})$ & 173.05 & 138.47 & 89.06 \\
$(\mathbf{e})$ & 178.79 & 143.09 & 92.06 \\
Closure time $\left(t_{C}\right)$ & 178.93 & 143.23 & 92.19 \\
\hline \hline
\end{tabular}



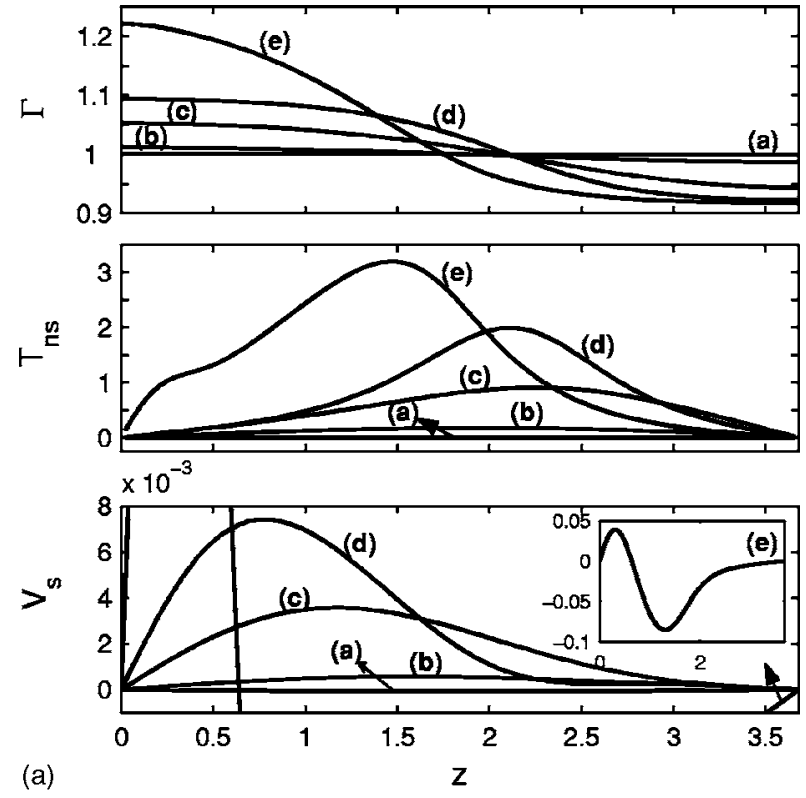

(a)
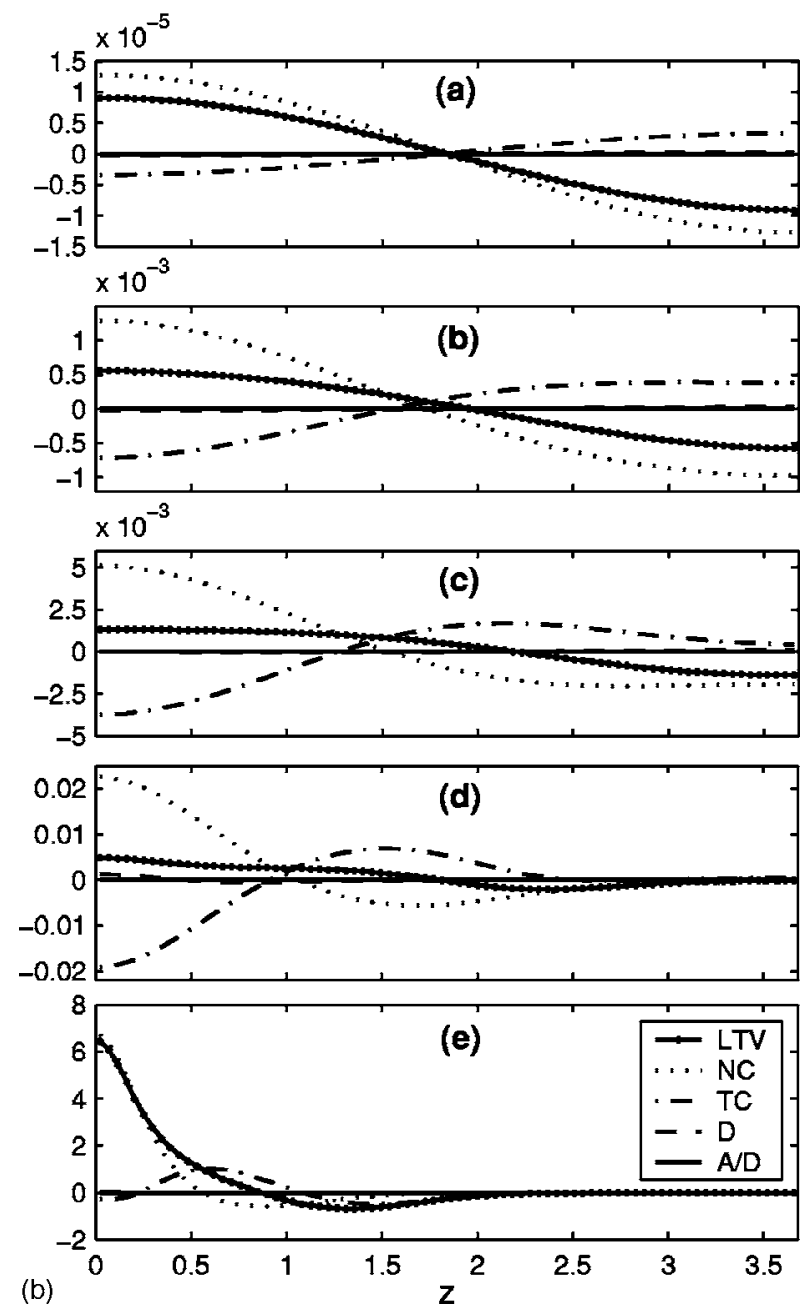

FIG. 11. (a) Profiles of the interfacial variables for the five stages shown in Fig. 10 for the RC with $K \rightarrow \infty$. From top to bottom: surfactant concentrations, tangential stresses, and tangential velocities. (b) Profiles, for the RC with $K \rightarrow 0$, of the five terms contributing to the interfacial mass balance [Eq. (31)]: local time variation (LTV), normal convection (NC), tangential convection (TC), diffusion (D), and adsorption/desorption (A/D). From top to bottom: the five stages [(a)-(e)] shown in Fig. 10. velocity. Each variable is shown for the five stages pictured in Fig. 10. It must be noticed that none of these variables presents any evidence of change at the former steps of the process, as proved by the curves denoted by (a). The reason is the slow progress of the instability as a consequence of the weak driving force acting at the beginning. On the other hand, near the final steps of the process the speed of the evolution is so large that some of the curves denoted by (e) go out of scale. This acceleration can be explained by examining the interfacial shapes shown in Fig. 10. The instability begins draining liquid from the right side of the domain toward the left and, as a consequence, the thickness of the liquid film decreases on that region while a liquid lobe develops at $z=0$. The large viscous forces arising in the thin film during the draining process restrain the growing speed of the lobe. However, a situation is reached where the draining process diminishes [stage (d)]. Near the configuration denoted by (e) the volume of liquid in the lobe is large enough to close the air gap and the motion of liquid in the neck is very slow-a fact that is clearly indicated when the streamlines are portrayed (see Campana et al. ${ }^{26}$ ). At this point, with a second small lobe becoming evident on the right side, the governing mechanisms of the instability change and the driving force provided by capillary pressure is just resisted by the weak forces (inertial and viscous) acting in the large lobe. The equilibrium between the driving and resisting forces is so out of balance that it cannot be altered by varying the surfactant solubility; therefore, the growing rate of the lobe at $z=0$ becomes very large and almost independent of $K$. This fact is confirmed by the results shown in the last row of Table $\mathrm{V}$, which indicate that in all three cases the stage (e) is about 0.13-0.14 time units away from closure. Consequently, since we wish to study how the solubility of the surfactant is related to the growing rate of the instability, we will focus our attention on the sequence of stages $(\mathbf{a})-(\mathbf{d})$.

The case of an insoluble surfactant $(K \rightarrow \infty)$ is examined first. Since the perturbation imposed onto the initially horizontal film [see Eq. (4)] generates a liquid motion in the bulk from right to left, one expects the solute to be transported by convection in the same manner. Figure 11(a) seems to confirm this presumption showing curves of $\Gamma$ whose left and right ends are above and below the initial concentration, respectively. However, we will immediately see that the solute moves from left to right.

On the left side of the domain, $\Gamma$ increases as the instability advances while the opposite occurs on the right side. The interfacial shear stress distribution, which is also shown in Fig. 11(a), is a direct consequence of the interfacial concentration gradients, originated by the distribution of surfactant just described. According to Eqs. (6) and (20), the interfacial shear stresses-or Marangoni stresses-are positive since the concentration gradients are negative; thus, by trying to drag the interface toward increasing values of arc length, these interfacial stresses oppose the action of the bulk which is attempting to move it toward $z=0$. Actually, in the present case $(K \rightarrow \infty)$, the Marangoni stresses overcome the forces originated in the bulk motion and the interfacial tangential velocities, though small, are positive, as Fig. 11(a) indicates. 

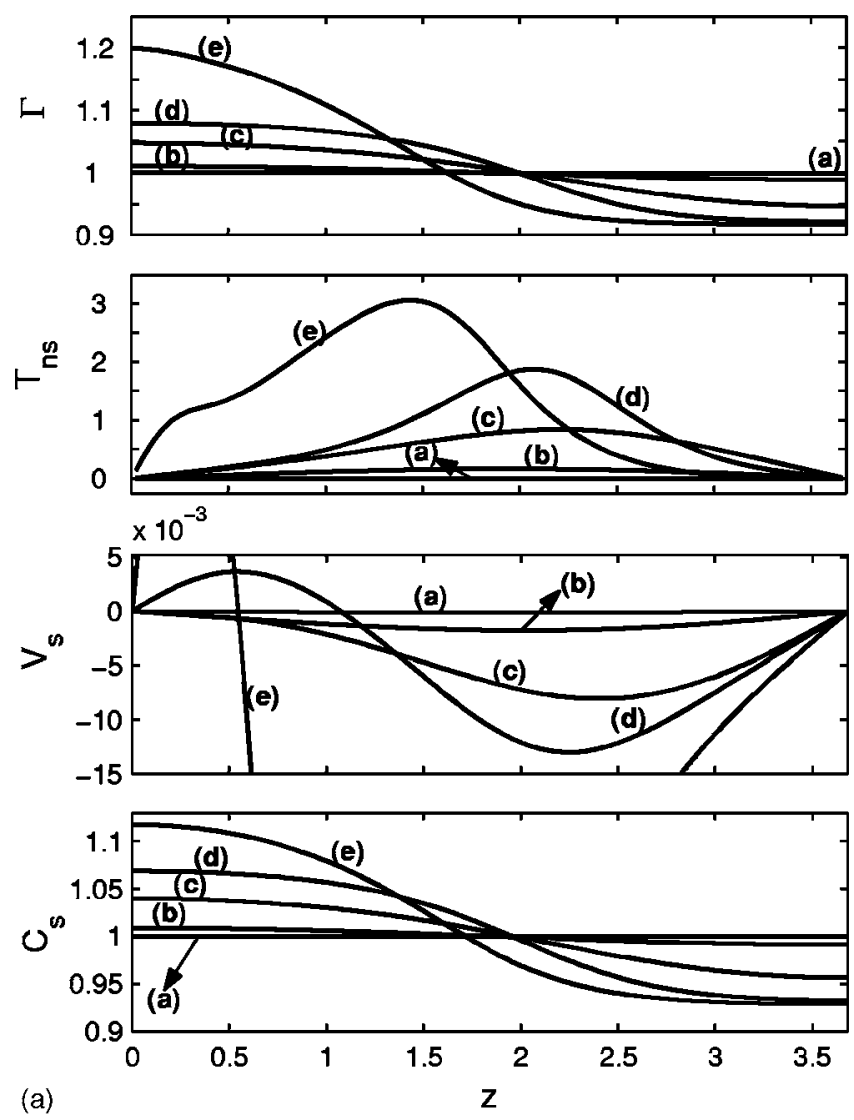
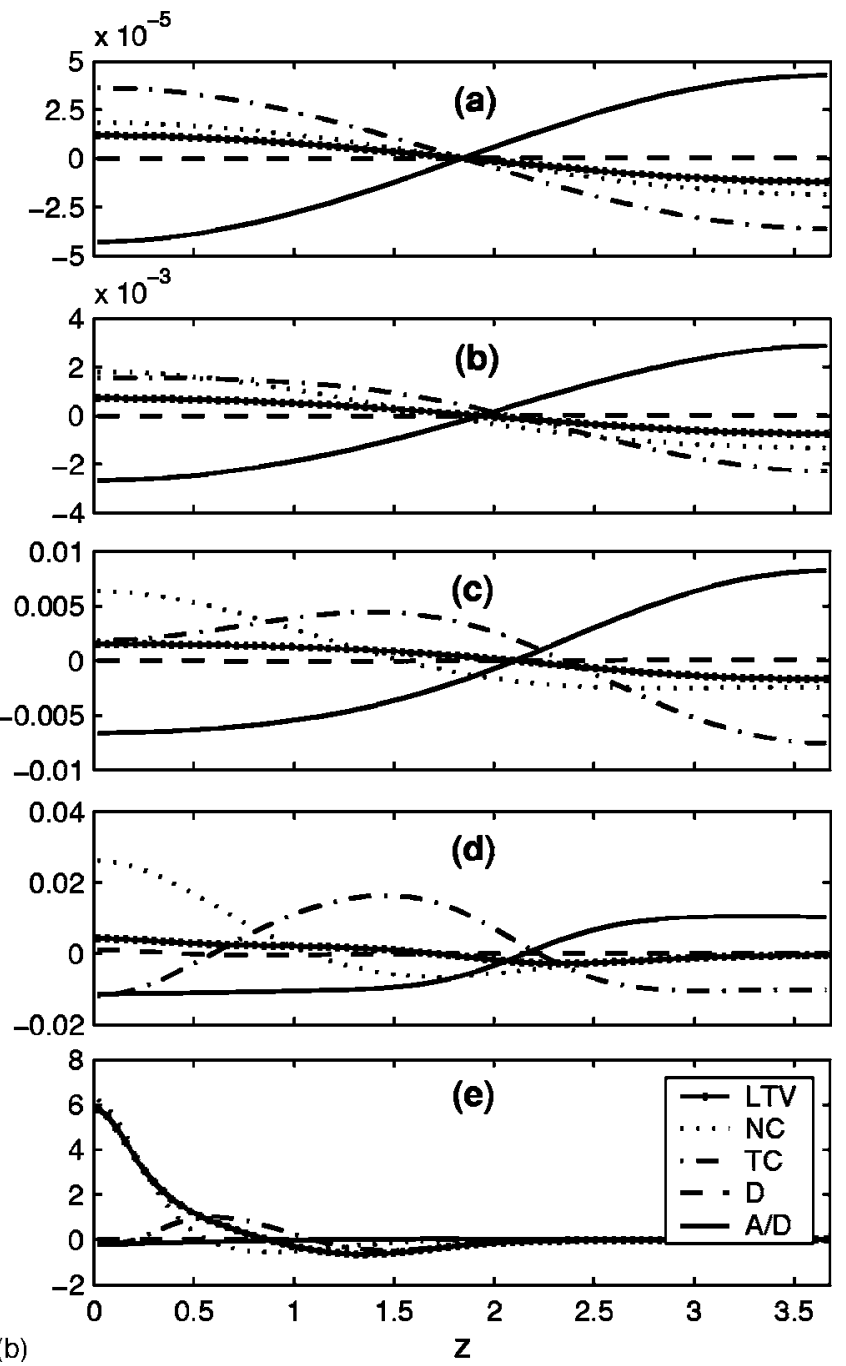

(b)

Z

FIG. 12. (a) As in Fig. 11(a) with $K=0.01$, and the bulk concentration of surfactant just at the interface $\left(C_{S}\right)$. (b) As in Fig. 11 (b) with $K=0.01$.

We have just observed for $K \rightarrow \infty$ that the tangential interfacial velocities are positive; then, convection must transport the solute adsorbed at the interface from left to right. Since interfacial diffusion should carry solute toward regions depleted of it, and there is no transport by adsorption/ desorption because the surfactant is insoluble, the question is by which mechanisms the concentration of surfactant increases on the left side of the domain and decreases at the opposite side. The answer is clearly given by the only term that is left in the interfacial balance equation, that is, the normal convection term that accounts for the interfacial stretching or shrinking. It is evident that near $z=0$, where the liquid lobe develops, the radius of the interface decreases and the extent of the interfacial surface does so at a quadratic rate; this process rapidly increases the concentration of surfactant there. The opposite occurs at the other end of the domain.

Figure 11(b) portrays, for the stages (a)-(e) shown in Fig. 10, the values presented by the five terms contributing to the interfacial balance equation when $K \rightarrow \infty$. The different scales employed in the vertical axis for the successive configurations observed, i.e., from (a) to (e), illustrate at once how the magnitude of the transfer process is changing as the instability develops. These results indicate that the contribution of diffusion to the interfacial mass balance is negligible at all stages, and naturally, the transport by sorption is zero; thus, the local time variation of surfactant concentration is balanced by both tangential and normal convection.

Results shown in Fig. 11(b) support our previous assertions: on the left side of the domain and near $z=0$, the tangential convection term diminishes the surfactant concentration while the opposite occurs with the normal convection term. Also, from stages (a) to (d), the local concentration of solute increases because the magnitude of the normal convection is somewhat larger than the magnitude of the tangential one. However at (e), when the process of closure has already been triggered, the picture completely differs from previous stages. At this point, normal convection has become the only term that counts, suggesting that a different mechanism is governing the unstable evolution.

Results for a slightly soluble surfactant are portrayed in Figs. 12(a) and 12(b). Figure 12(a) shows curves of interfacial concentration of surfactant that are quite similar to those shown in Fig. 11(a); their shapes are sigmoid too, but in this 

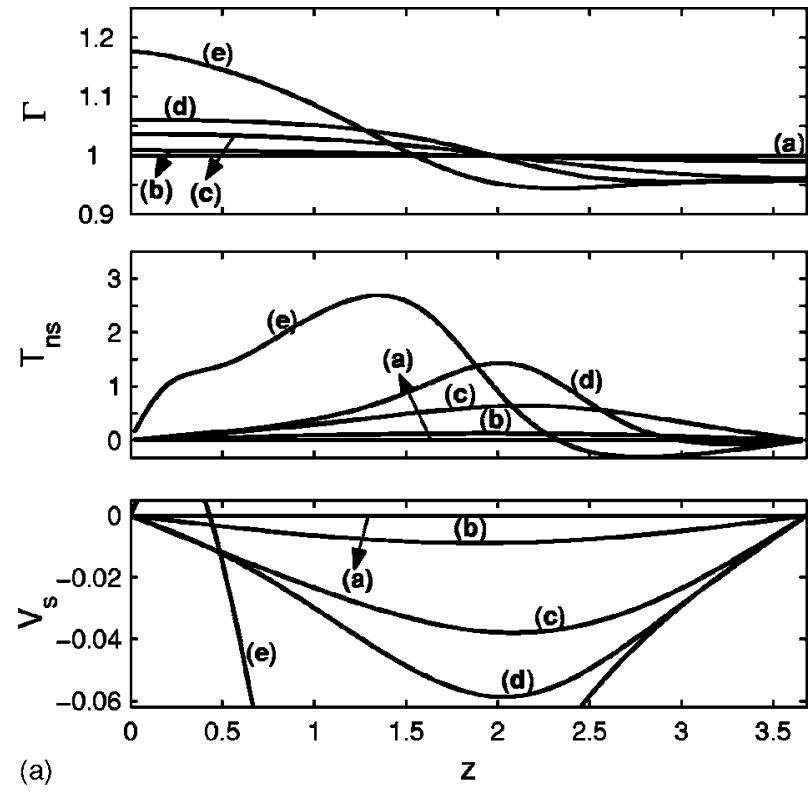

(a)
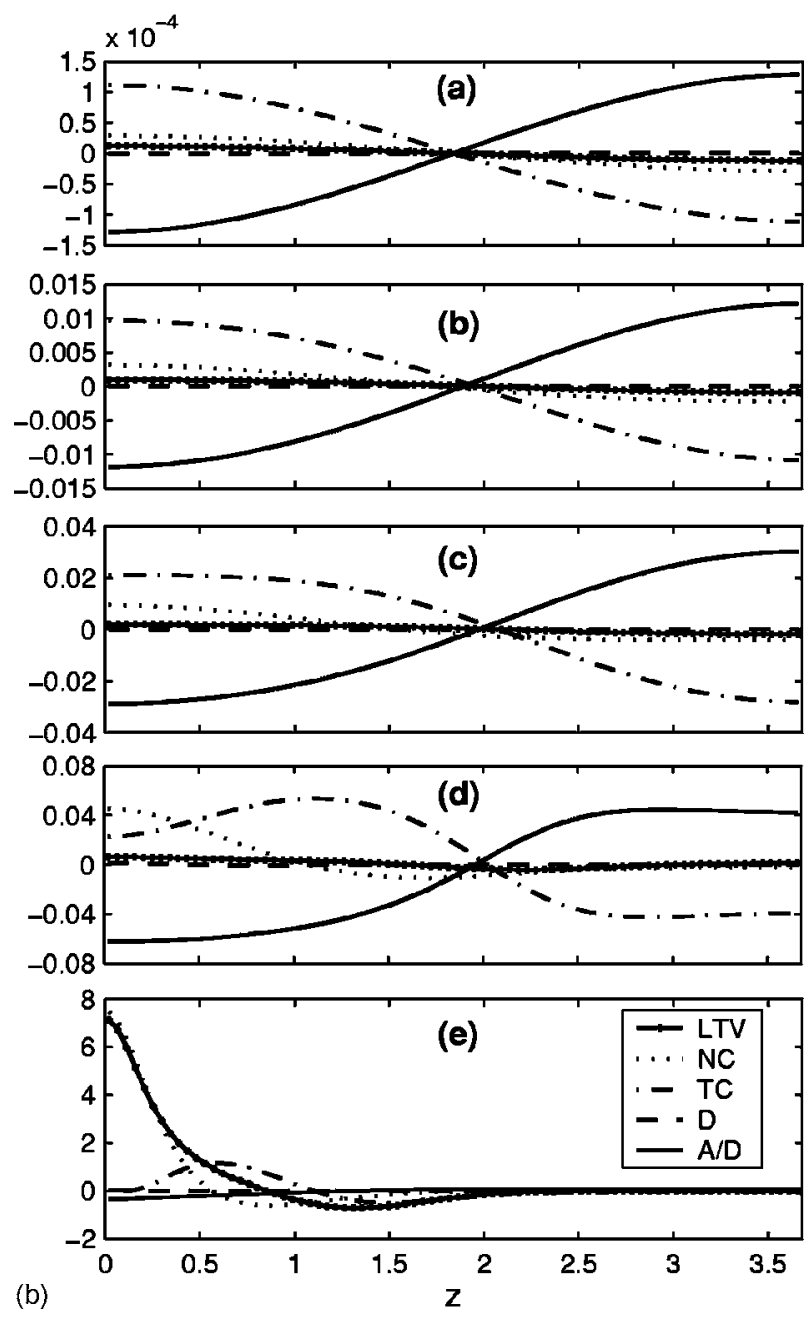

FIG. 13. (a) As in Fig. 11(a) with $K \rightarrow 0$. (b) As in Fig. 11(b) with $K \rightarrow 0$.

case part of the solute at the interface is transferred to the bulk. This feature is evidenced by the profiles of concentration of surfactant at the subsurface $\left(C_{S}\right)$.

In Fig. 12(a), the values of $\Gamma$ are, on average, slightly closer to one than in Fig. 11(a); i.e., the sigmoid shapes are now somewhat gentler and the concentration gradients are slightly smaller than before. Obviously, these new characteristics should in turn produce changes in the interfacial shear stresses and then in the interfacial tangential velocities too. Though the curves of interfacial shear stresses are very similar to those shown in Fig. 11(a), their values are barely smaller and these small differences produce a drastic change in the interfacial velocities. Figure 12(a) exhibits negative interfacial velocities - except near the left end of the domain and just for the final stages of the instability.

The just mentioned change of sign in the velocity and the transfer of solute toward the bulk phase are the new features that entirely modify the way the interfacial mass balance [Eq. (31)] is accomplished. From the former steps of the instability, the interfacial tangential velocity convects solute toward $z=0$ as Fig. 12(b) shows. Near $z=0$ the added contribution of the two convection terms is almost counterbalanced by desorption, while the rate of variation of the interfacial concentration of surfactant makes up for the difference. Again, as in the insoluble case shown in Fig. 11(b), the diffusive terms are negligible.

When the unstable process advances, the interfacial gradients of $\Gamma$ become larger and so do the elastic effects that, at a certain point between stages $(\mathbf{c})$ and $(\mathbf{d})$, reverse the sign of the tangential interfacial velocity near $z=0$. At stage (d), the interfacial velocity simultaneously presents a relative minimum and a relative maximum; this characteristic induces negative values of tangential convection at both ends of the domain and positive values in between (see stage (d) in Fig. 12(b)). Finally, when the unstable process is almost finishing [stage (e)], the picture portrayed in Fig. 12(b) turns out to be almost identical to that shown in Fig. 11(b); this similitude suggests that the mechanisms governing the final stages of the instability do not depend on the solubility of the solute.

Figures 13(a) and 13(b) present the results for a surfactant that is totally soluble in the liquid phase (i.e., $K \rightarrow 0$ ); since in this case $C=C_{S}=1$, the driving force for the sorption process should be larger than in the case just analyzed with $K=0.01$. Then, we expect to see a distribution of $\Gamma$ closer to 1 and smaller concentration gradients. Figure 13(a) confirms that expectation and it also shows curves of interfacial shear stresses that are clearly weaker than in previous cases. Actually, at stage (d), the curve of $\Gamma$ presents a minimum when $z$ is approximately 2.9; consequently, between that point and the right end of the domain, the values of $T_{n s}$ are negative.

As the elastic effects weaken, the bulk motion becomes the dominant driving force for the interface; Fig. 13(a) shows negative values of the tangential interfacial velocities with magnitudes that in general are four/five times larger than in Fig. 12(a). The term accounting for the tangential convection of solute also increases significantly as Fig. 13(b) indicates; from stages (a) to (c) and near $z=0$, this term and the mass transport by sorption prevail in the interfacial mass balance. Finally, when stage (d) is about to be reached, the normal convection term becomes important and surpasses tangential convection. Again, as in the cases previously seen, stage (e) does not appear to depend on surfactant solubility.

In this section, we have shown how the solubility of 

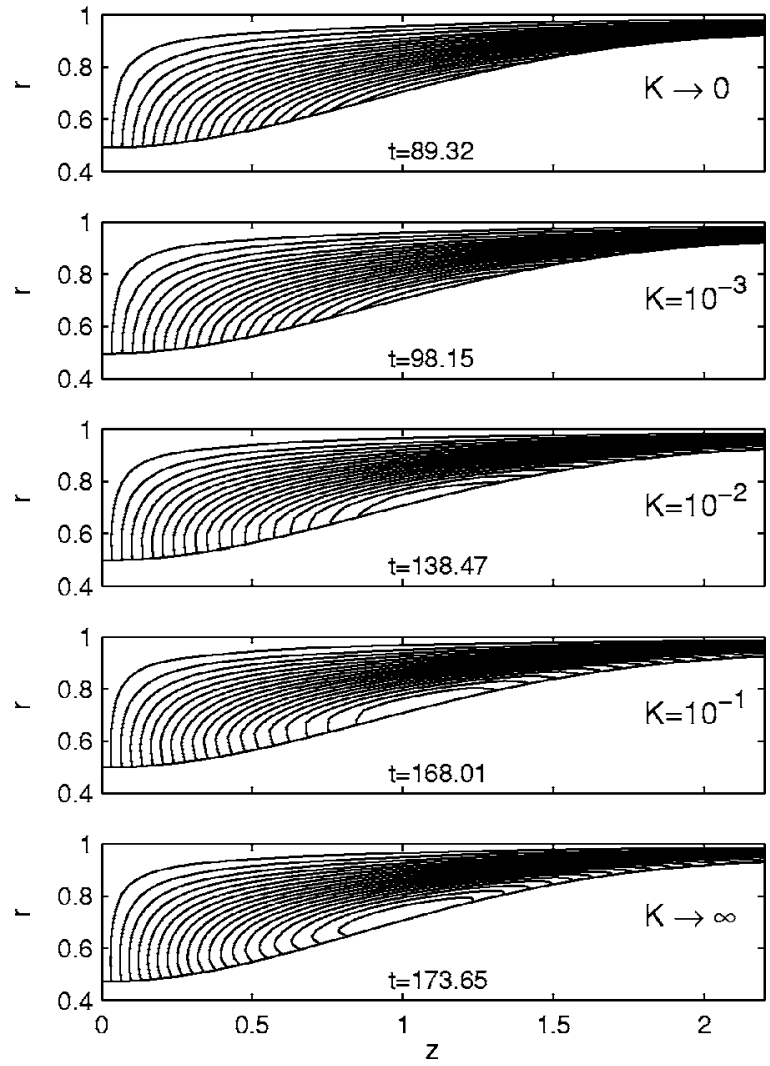

FIG. 14. Streamlines at stage (d) of Fig. 10 for the RC and several values of surfactant solubility.

surfactants affects the terms of the interfacial mass balance equation; these changes in the mass transport terms also induce changes in the interfacial variables, particularly in the interfacial velocities, which ends up in the already shown closure time variations. However, not only are the interfacial variables affected, but also both the flow field and the concentration field in the neighborhood of the interface are affected.

Figure 14 portrays the streamlines of five systems whose dimensionless parameters are those of the RC, but they differ in the solubility of the surfactant. The five systems are at the same stage of the instability process while the solubility considered ranges from totally soluble (CCB) to totally insoluble. Clearly, near the gas-liquid interface, the streamlines are noticeably affected when the surfactant is insoluble; in that case, the Marangoni stresses are the largest, and the interface moves into the opposite direction to the bulk motion dragging the adjacent liquid layers. This behavior gives rise to a small eddy located close to the interface and near $z=1$, a feature that can easily be envisaged from the streamlines shown in Fig. 14. As the solubility increases, the Marangoni stresses diminish, and a point is reached where the bulk motion takes over and the tangential interfacial velocity becomes negative.

The concentration fields in the bulk phase are not shown since they result as expected; i.e., the isoconcentration lines are almost parallel to the interface and the concentration gradients are much larger in the normal direction to the interface than along it.

\section{CONCLUDING REMARKS}

This work is the sequel of a previously published one $\left(\right.$ Campana et $\mathrm{al.}^{26}$ ) where the present model-restricted to insoluble surfactants-was validated and was employed to study the Rayleigh instability in capillaries under the presence of insoluble surfactants. Here, after extending the capabilities of our model by including both sorption transport and bulk mass transport, we have broadened those analyses by considering surfactants with different degrees of solubility. The goal of the present paper was to disclose the influence that surfactant solubility might have on the evolution of this instability, and in particular on the time needed for its completion.

We confirmed the results of previously performed numerical analyses: surfactants do not change the stability of the system; however, they slow down the unstable evolutions and closure times might turn out to be up to four/five times longer. ${ }^{23}$ More recently, Kwak and Pozrikidis ${ }^{25}$ pointed out essentially the same issue when they showed that surfactants do not alter the successive shapes adopted by the interface but the growth rate of the interfacial waves, which is considerably delayed.

The predictions we obtained showed that the retardation effects produced by surfactants diminish as their solubility increases. This outcome was expected; however, the magnitude of the relative changes in closure times, caused by surfactants of relatively low solubility, was not. In fact, the results presented for our reference case showed retardation effects of about $74 \%$ for $\beta=0.1$ and $29 \%$ for $\beta=0.01$, with regard to the values produced by the corresponding insoluble surfactant. These predictions about Rayleigh instability suggest that careful considerations must be given to surfactant solubility when processes governed by interfacial dynamics are analyzed.

The numerical technique, formerly employed for solving free surface flow with insoluble surfactants, has been successfully extended to cope with soluble ones. This extension required the addition of the governing equations for interfacial sorption and for mass transport in the bulk, to an already complex code that deals with transient free surface flows, locally varying interfacial properties, and interfacial mass transport. The results obtained are instructive because they illustrate how the transport terms in the interfacial mass balance change as the instability advances, and how they change when the solubility of surfactants is varied.

The present analysis is restricted to the case of rather dilute solutions; thus, our assumption of a linear relationship for the surface equation of state is acceptable. Nonetheless, more realistic approximations should be examined.

\section{ACKNOWLEDGMENTS}

Universidad Nacional del Litoral, CONICET, and ANPCyT provided financial support for this work.

${ }^{1}$ C.-W. Park and G. M. Homsy, "Two-phase displacement in Hele Shaw cells: Theory,” J. Fluid Mech. 139, 291 (1984).

${ }^{2}$ D. A. Reinelt and P. G. Saffman, "The penetration of a finger into a viscous fluid in channel and tube," SIAM (Soc. Ind. Appl. Math.) J. Sci. Stat. Comput. 6, 542 (1985). 
${ }^{3}$ L. W. Schwartz, H. M. Princen, and A. D. Kiss, "On the motion of bubbles in capillaries tubes," J. Fluid Mech. 172, 259 (1986).

${ }^{4}$ J. Ratulowski and H. C. Chang, "Marangoni effects of trace impurities on the motion of long bubbles in capillaries," J. Fluid Mech. 210, 303 (1990).

${ }^{5}$ M. J. Martinez and K. S. Udell, "Boundary integral analysis of the creeping flow of long bubbles in capillaries," Trans. ASME, J. Appl. Mech. 56, 211 (1989).

${ }^{6} \mathrm{M}$. D. Giavedoni and F. A. Saita, "The axisymmetric and plane cases of a gas phase steadily displacing a Newtonian liquid. A simultaneous solution of the governing equations," Phys. Fluids 9, 2420 (1997).

${ }^{7}$ M. Severino, M. D. Giavedoni, and F. A. Saita, "A gas phase displacing a liquid with soluble surfactants out of a small conduit: The plane case," Phys. Fluids 15, 2961 (2003).

${ }^{8}$ S. N. Ghadiali and D. P. Gaver III, "The influence of non-equilibrium surfactant dynamics on the flow of a semi-infinite bubble in a rigid cylindrical capillary tube," J. Fluid Mech. 478, 165 (2003).

${ }^{9}$ M. Severino, D. M. Campana, and M. D. Giavedoni, "Effects of a surfactant on the motion of a confined gas-liquid interface. The influence of the Peclet number," Lat. Am. Appl. Res. 35, 225 (2005).

${ }^{10}$ F. Fairbrother and A. Stubbs, "Studies in electroendosmosis. Part VI. The bubble-tube method of measurements," J. Chem. Sci. 1, 527 (1935).

${ }^{11}$ F. P. Bretherton, "The motion of long bubbles in tubes," J. Fluid Mech. 10, 166 (1961).

${ }^{12}$ Lord Rayleigh, "On the capillary phenomena in jets. Appendix I," Proc. R. Soc. London 29, 71 (1879).

${ }^{13}$ Lord Rayleigh, "On the instability of a cylinder of viscous liquid under capillary force," Philos. Mag. 34, 145 (1892)

${ }^{14} \mathrm{C}$. Weber, "Zum zerfall eines ussigkeitsstrahles," Z. Angew. Math. Mech. 11, 136 (1931)

${ }^{15} \mathrm{~S}$. Tomotika, "On the instability of a cylindrical thread of a viscous liquid surrounded by another viscous liquid," Proc. R. Soc. London, Ser. A 150, $322(1935)$

${ }^{16}$ S. Goren, "The instability of an annular thread of fluid," J. Fluid Mech. 12, 309 (1962).

${ }^{17}$ H. L. Goldsmith and S. G. Mason, "The flow of suspensions through tubes. II-Single large tubes," J. Colloid Sci. 18, 237 (1963).

${ }^{18}$ P. S. Hammond, "Nonlinear adjustment of a thin annular film of viscous fluid surrounding a thread of another within a circular cylindrical pipe," J. Fluid Mech. 137, 363 (1983).

${ }^{19}$ P. A. Gauglitz and C. J. Radke, "An extended evolution equation for liquid film breakup in cylindrical capillaries," Chem. Eng. Sci. 43, 1457 (1988).

${ }^{20}$ M. Johnson, R. D. Kamm, L. W. Ho, A. Shapiro, and T. J. Pedley, "The nonlinear growth of surface tension driven instabilities of a thin annular film," J. Fluid Mech. 223, 141 (1991).
${ }^{21}$ D. Halpern and J. B. Grotberg, "Fluid-elastic instabilities of liquid-lined flexible tubes," J. Fluid Mech. 244, 615 (1992).

${ }^{22}$ D. Halpern and J. B. Grotberg, "Surfactant effects on fluid-elastic instabilities of liquid-lined flexible tubes: A model of airway closure," J. Biomech. Eng. 115, 271 (1993).

${ }^{23}$ D. R. Otis, M. Johnson, T. J. Pedley, and R. D. Kamm, "The role of pulmonary surfactant in airway closure," J. Appl. Physiol. 59, 1323 (1993).

${ }^{24}$ K. J. Cassidy, D. Halpern, B. G. Ressler, and J. B. Grotberg, "Surfactant effects in model airway closure experiments," J. Appl. Physiol. 87, 415 (1999).

${ }^{25}$ S. Kwak and C. Pozrikidis, "Effects of surfactants on the instability of a liquid thread or annular layer. Part I: Quiescent fluids," Int. J. Multiphase Flow 27, 1 (2001).

${ }^{26}$ D. M. Campana, J. Di Paolo, and F. A. Saita, "A 2-D model of Rayleigh instability in capillary tubes. Surfactant effects," Int. J. Multiphase Flow 30, 431 (2004).

${ }^{27}$ H. Khesghi and L. E. Scriven, "Penalty finite element analysis of unsteady free surface flows," Finite Elements in Fluids 5, 393 (1984).

${ }^{28}$ M. D. Giavedoni, "A numerical study of the two dimensional dynamic behavior of a thin liquid film subject to a vertical oscillation," Ind. Eng. Chem. Res. 34, 356 (1995).

${ }^{29}$ D. A. Edwards, H. Brenner, and D. T. Wasan, Interfacial Transport Processes and Rheology, Series in Chemical Engineering (ButterworthHeinemann, Boston, 1991).

${ }^{30} \mathrm{H}$. Wong, D. Rumschitzki, and C. Maldarelli, "On the surfactant mass balance at a deforming fluid interface," Phys. Fluids 8, 3203 (1996).

${ }^{31}$ M. D. Giavedoni and F. A. Saita, "The axisymmetric and plane cases of a gas phase steadily displacing a Newtonian liquid. A simultaneous solution of the governing equations," Phys. Fluids 9, 2420 (1997).

${ }^{32}$ M. A. Crisfield, "A fast incremental iterative solution procedure that handles 'snap-through'," Comput. Struct. 13, 55 (1981).

${ }^{33}$ P. M. Gresho, R. L. Lee, and R. L. Sani, in Recent Advances in Numerical Methods in Fluids, edited by C. Taylor and K. Morgan (Pineridge, Swansea, UK, 1980), Vol. 1, Chap. 2, pp. 27-79.

${ }^{34}$ C. M. Corvalan and F. A. Saita, "Automatic step-size control in continuation procedures," Comput. Chem. Eng. 5, 729 (1991).

${ }^{35}$ S. Balay, K. Buschelman, V. Eijkhout, W. D. Gropp, D. Kaushik, M. G. Knepley, L. Curfman McInnes, B. F. Smith, and H. Zhang, "PETSc Users Manual," Report ANL-95/11-Revision 2.1.5, Argonne National Laboratory, 2004

${ }^{36} \mathrm{C}-\mathrm{H}$. Chang and E. I. Franses, "Adsorption dynamics of surfactants at the air/water interface: A critical review of mathematical models, data and mechanisms," Colloids Surf., A 100, 1 (1995). 